Portland State University

PDXScholar

Civil and Environmental Engineering

Undergraduate Honors Theses

Spring 2021

\title{
Distributionally Robust Optimization Utilizing Facility Location Problems
}

\author{
Elijah Kling \\ Portland State University
}

Follow this and additional works at: https://pdxscholar.library.pdx.edu/cengin_honorstheses

Part of the Civil Engineering Commons, and the Transportation Engineering Commons Let us know how access to this document benefits you.

\section{Recommended Citation}

Kling, Elijah, "Distributionally Robust Optimization Utilizing Facility Location Problems" (2021). Civil and Environmental Engineering Undergraduate Honors Theses. 12.

https://doi.org/10.15760/honors.1147

This Thesis is brought to you for free and open access. It has been accepted for inclusion in Civil and Environmental Engineering Undergraduate Honors Theses by an authorized administrator of PDXScholar. Please contact us if we can make this document more accessible: pdxscholar@pdx.edu. 
DISTRIBUTIONALLY ROBUST OPTIMIZATION UTILIZING

FACILITY LOCATION PROBLEMS

BY

Elijah Kling

A thesis submitted in partial fulfillment

of the requirement for the degree of

\section{BACHELOR OF SCIENCE}

IN

CIVIL AND ENVIRONMENTAL ENGINEERING

Thesis Advisor:

Dr. Avinash Unnikrishnan

Portland State University

(C)2021 


\section{ACKNOWLEDGMENTS}

I extend a huge thank you to Dr. Avinash Unnikrishnan, whose guidance and brilliance made this work possible.

I would like to thank Darshan Chauhan, who provided the tools and examples which allowed for this thesis to be completed.

I would also like to thank the National Science Foundation Grant number 1826337 "Collaborative Research: Real-Time Stochastic Matching Models for Freight Electronic Marketplace" for supporting this research.

Lastly, I would like to thank my instructors, professors, and peers for creating a unique and motivating learning environment. 


\begin{abstract}
Facility location problems are a used in widespread application in transportation, freight, supply chain, and logistics problems. Models can be developed as deterministic, where all parameters are known, or robust, where a parameter has uncertainty. This thesis explores a new method for developing robust formulation and compares the implications of assuming values for this uncertain parameter. Two models are solved, and both are compared against their deterministic counterparts using numerical analysis. By manipulating the input parameters and considering real world implications of the solutions, either the robust or deterministic can show better performance.
\end{abstract}




\section{TABLE OF CONTENTS}

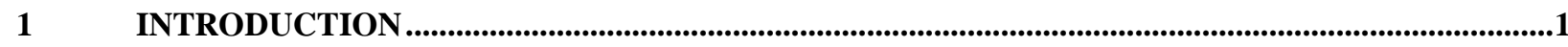

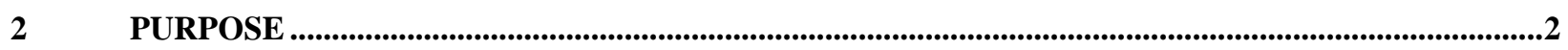

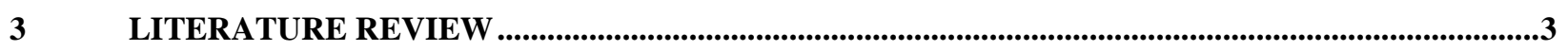

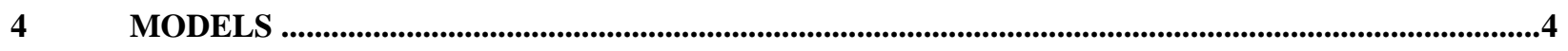

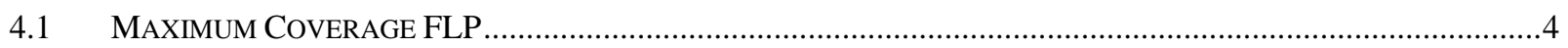

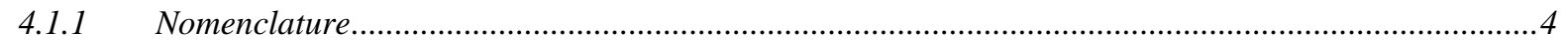

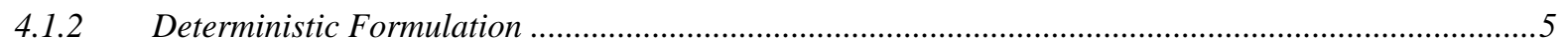

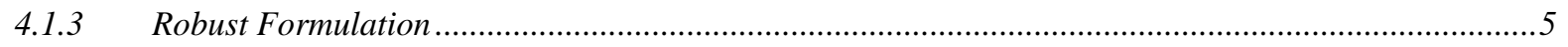

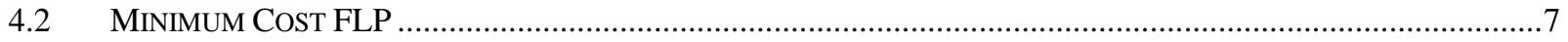

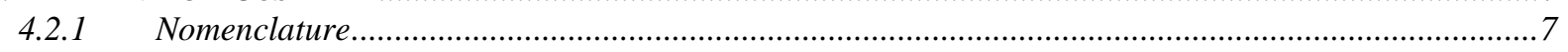

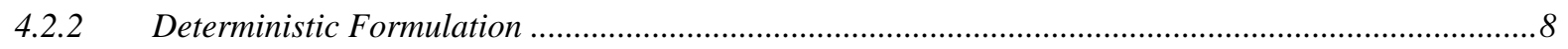

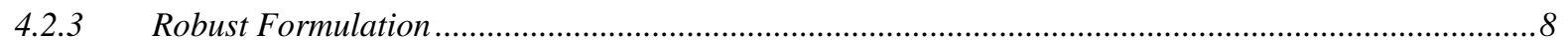

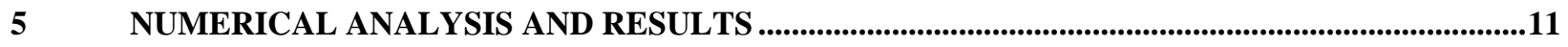

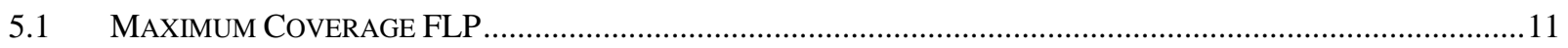

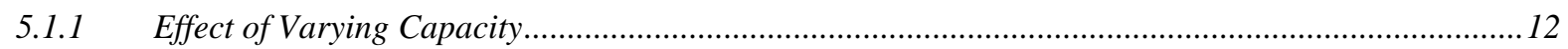

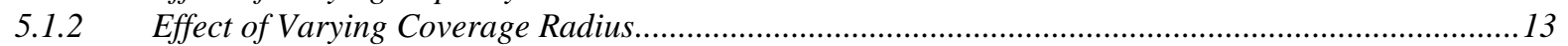

5.1.3 Effect of Varying Facilities Located ................................................................................................ 14

5.1.4 Comparing Symmetric and Various Asymmetric Distributions ........................................................16

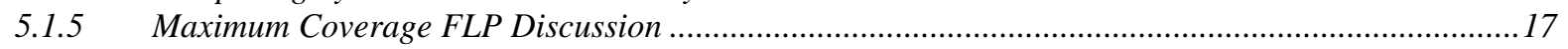

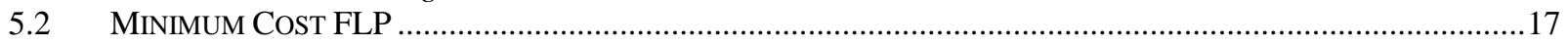

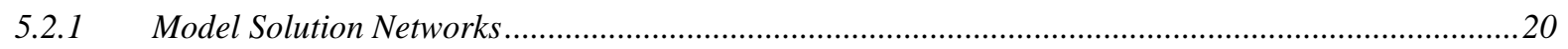

5.2.2 MCS Results Considering Symmetric and Asymmetric Distributions ..............................................21

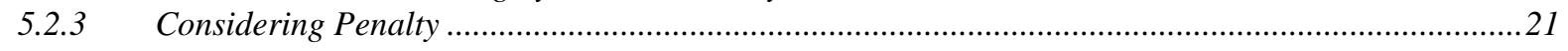

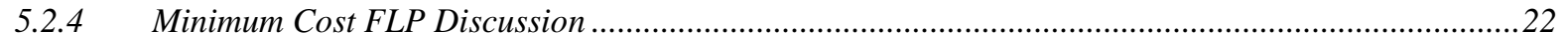

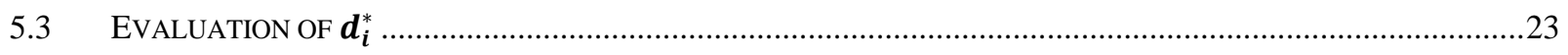

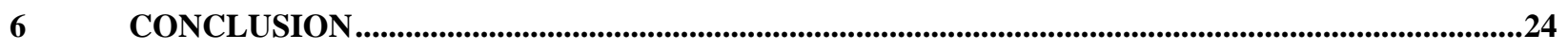

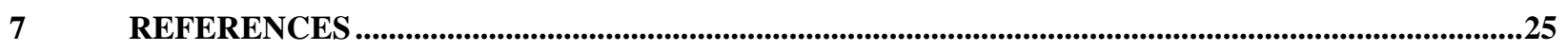

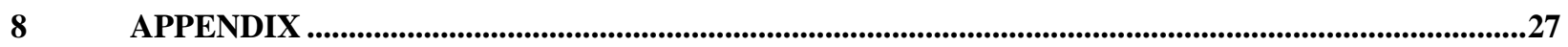

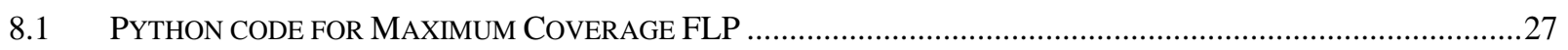

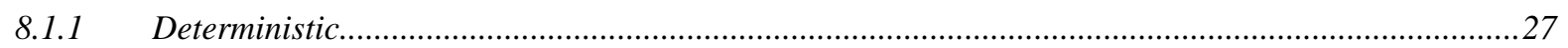

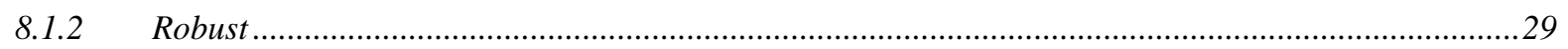

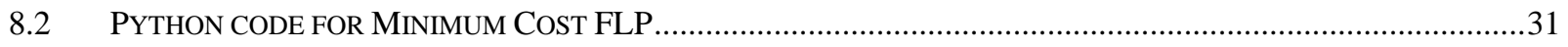

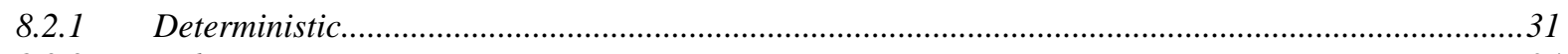

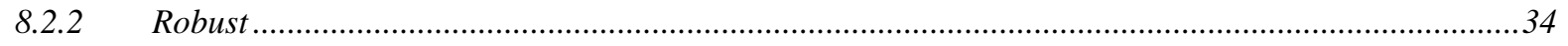




\section{LIST OF TABLES}

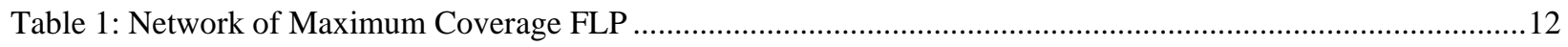

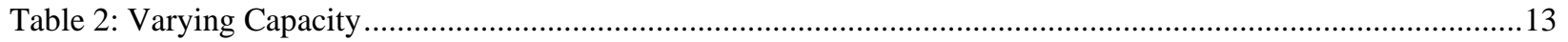

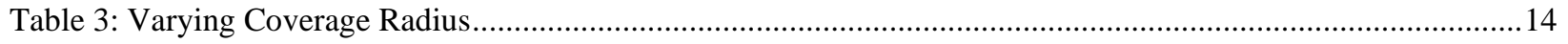

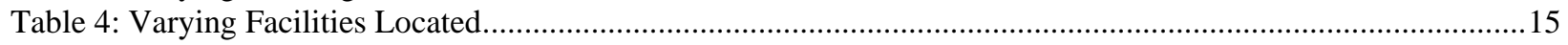

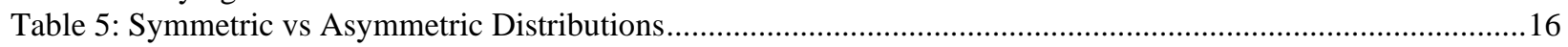

Table 6: Minimum Cost FLP Network (Demands) ……................................................................................. 18

Table 7: Minimum Cost FLP Network (Capacities and Fixed Costs) ……...........................................................18

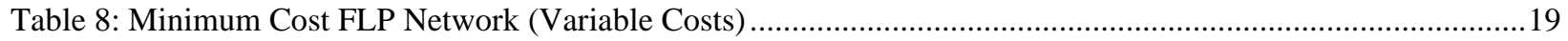

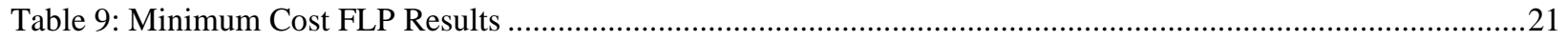

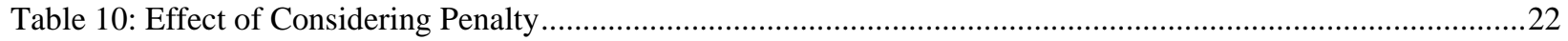

\section{LIST OF FIGURES}

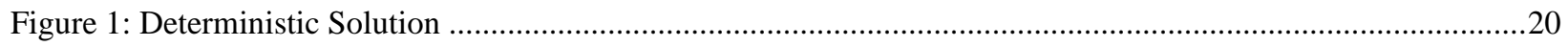

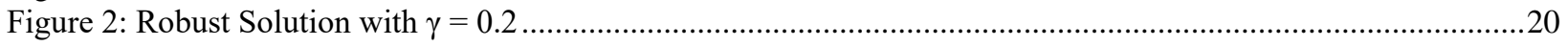

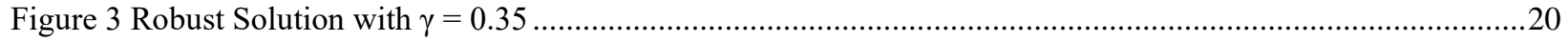




\section{INTRODUCTION}

Transportation, supply chain, and freight systems are typically designed through maximizing efficiency. This paper will primarily focus on facility location problems (FLP), which have widespread application. Facility is used as a broad term including but not limited to factories, seaports, schools, public transit stops, and more. A maximum coverage FLP is used in public sector applications to locate facilities by maximizing the demand served. Examples of public sector applications include locating post offices, health clinics, ambulances, fire departments, etc. FLPs are also used in the private sector. Private sector applications will often use a cost minimization perspective as maximizing coverage is not essential.

The FLPs which will be considered in this paper will include one public and one private sector application. The problems are initially deterministic, meaning that no randomness is involved to develop a solution. Each constraint which is accounted for in the problem is known, unchanging, and not affected by chance. Using deterministic problems as a basis for development poses concerns because many deterministic constraints are not a reality.

A robust problem will account for uncertainty. There are multiple ways to achieve this. For our purposes, one parameter will be assumed to have a distribution with defined bounds. Using the methods from (Ghosal and Wiesemann, 2020), an equivalent parameter will be generated to encapsulate the uncertain parameter. The goal of this paper will be to create robust models which are computationally quicker and easier to solve. 


\section{PURPOSE}

An important theme in civil engineering problems is how much do we design for? That is, how can we be certain that a design will meet the criteria without using too many resources, effort, and labor? Designing to meet an average doesn't work because failure occurs $50 \%$ of the time. Designing something to never fail is incredibly costly and overengineered. Canon 1 of The American Society of Civil Engineers (ASCE) Code of Ethics states that "Engineers shall hold paramount the safety, health, and welfare of the public and shall strive to comply with the principles of sustainable development in the performance of their professional duties." (Code of Ethics)

Above all else, a design should be safe and healthy to use. The most important criterion after that is welfare of the public. The problem we are evaluating becomes clearer. When dealing with uncertainty, we must be able to quantify what can be expected. From there we can ensure that whatever we are designing for captures safety, health, and welfare of the public. When choosing how to locate facilities, it is important to ensure that a decision maker can see how the uncertainty is quantified. By creating a stochastic model, the certainty for which a constraint will be satisfied can be controlled. For the purposes of this research, demand is considered as uncertain. Uncertain demand will be implemented into two facility location models to show how well the stochastic solution meets the objectives. To provide a reasonable design, the uncertain demand should be permitted to exceed the capacity of the facility a very small fraction of the time. In both models this is less than five percent of the time. A theme of this paper is to obtain a practical robust model which provides better performance than its deterministic counterpart. 


\section{LITERATURE REVIEW}

A plethora of work has been conducted on FLPs. (Church and ReVelle, 1974) outlines the maximum coverage FLP which paved the road for the continuous development of problems in this field. This literature review aims to illustrate the diversity of application though the following relevant work. (Current et al., 2002) outlines the widespread application and usefulness of maximum coverage FLPs. A model from (Esnaf and Küçükdeniz, 2009) shows a broader method of locating facilities by eliminating the set of facilities to choose from. Instead, facilities are located anywhere to maximize demand. (Arabani and Farahani, 2012) show the dynamics between different minimum cost FLPs. (Chauhan et al., 2019) applies a maximum coverage FLP to dronedelivery and demonstrates how FLPs will be adapted to changing infrastructure. (Karatas and Dasci, 2020) shows how a FLP can incorporate more levels of facilities to maximize demand over an entire supply chain system. (Arslan, 2021) develops a solution considering the choice to locate a facility or route a vehicle. FLPs are incredibly diverse in their applications and continue to evolve with development.

Many works also consider uncertainty. (Snyder, 2006) reviews FLP problems under uncertainty and shows the diversity in objectives which have been developed. A study on linear optimization problems by (Bertsimas and Sim, 2004) shows how robust solutions may be too conservative. (Wang et al., 2002) shows algorithms considering $M / M / 1$ queueing systems met with stochastic demand. (Miranda and Garrido, 2004) utilize stochastic demand in their network design model aimed at incorporating short- and medium-term decisions. (Baron et al., 2011) and (Gülpınar et al., 2013) show various robust strategies for FLPs under uncertain demands. (Naoum-Sawaya and Elhedhli, 2013) present a stochastic optimization model applied to ambulance deployment. (Berglund and Kwon, 2014) present a robust FLP to minimize cost of hazardous waste transportation. (Lutter et al., 2017) explore robust solutions to set covering problems through studying mixed integer linear program problems. (Zhong et al., 2020) apply optimization to a facility location and vehicle routing problem. (Chauhan et al., 2020) extends the work of (Chauhan et al., 2019) and applies a robust optimization approach to an integer linear programming model. (Basciftci et al., 2021) considers stochastic demands for a two-stage decision-dependent optimization model. Considering uncertain parameters, particularly demand, is incredibly common in FLPs and other network optimization models. 


\section{MODELS}

The two models used to study the robust solution are a maximum coverage FLP and a minimum cost FLP. The robust models will be developed using a method proposed by (Ghosal and Wiesemann, 2020)

\subsection{Maximum Coverage FLP}

The objective of a maximum coverage FLP is in its name: to maximize the coverage. This type of FLP is more applicable in the case of public sector development. The model presented is capacitated and includes a coverage radius. For the purposes of maximizing infeasibility in numerical analysis, the model will be assumed to have an infinite coverage radius.

\subsubsection{Nomenclature}

Sets

I Set of demand points

$J \quad$ Set of potential facility locations

Indices

$i \in I$

$j \in J$

Parameters

$\varepsilon \quad$ Probability Parameter

$\gamma \quad$ Deviation Parameter

$a_{j} \quad$ Importance of meeting demand point $\mathrm{j}$

$\tilde{d}_{i} \quad$ Probabilistic demand of point $i$

$d_{i} \quad$ Nominal demand of point $i$

$U \quad$ Capacity

$s \quad$ Coverage radius

$L_{i j} \quad$ Distance between demand point and facility location

$M_{i j} \quad 1$ if $L_{i j} \leq s ; \infty$ otherwise

$p \quad$ Maximum number of facilities to locate

\section{Decision Variables}

$x_{j} \quad$ Binary: Equal to 1 if facility $i$ is opened; 0 otherwise 
$y_{i j} \quad$ Binary: Equal to 1 if demand point $i$ is serving facility $j ; 0$ otherwise

\subsubsection{Deterministic Formulation}

The objective is to maximize the coverage across all demand points (1).

maximize $\quad \sum_{i \in I} \sum_{j \in J} a_{i} y_{i j}$

S.To.

$$
\begin{aligned}
& \sum_{i \in I} M_{i j} d_{i} y_{i j} \leq U x_{j}, \quad \forall j \in J \\
& \sum_{j \in J} M_{i j} y_{i j} \leq 1, \quad \forall i \in I \\
& y_{i j} \leq x_{j}, \quad \forall i \in I, j \in J \\
& \sum_{j \in J} x_{j} \leq p \\
& x_{j} \in\{0,1\}, \quad \forall j \in J \\
& y_{i j} \in\{0,1\}, \quad \forall i \in I, j \in J
\end{aligned}
$$

(2) ensures that demand does not exceed capacity. Constraint (3) prevents a single demand point from mapping to more than one facility and (4) ensures that demand points are not mapped to closed facilities. (5) controls how many facilities are opened and (6-7) are variable definitions.

\subsubsection{Robust Formulation}

We begin with the deterministic problem. The nominal demand will now be considered as uncertain. Letting $\tilde{d}_{i}$ represent the uncertain demand, equation (8) is employed in place of (2) with a confidence of at least $1-\varepsilon$.

$$
\begin{gathered}
\operatorname{maximize} \quad \sum_{i \in I} \sum_{j \in J} a_{i} y_{i j} M_{i j} \\
\text { S.To. } P\left(\sum_{i \in I} M_{i j} \tilde{d}_{i} y_{i j} \leq U x_{j}\right) \geq 1-\varepsilon, \quad \forall j \in J, P \in \mathbb{P} \\
\sum_{j \in J} M_{i j} y_{i j} \leq 1, \quad \forall i \in I \\
y_{i j} \leq x_{j}, \quad \forall i \in I, j \in J \\
\sum_{j \in J} x_{j} \leq p
\end{gathered}
$$




$$
\begin{gathered}
x_{j} \in\{0,1\}, \quad \forall j \in J \\
y_{i j} \in\{0,1\}, \quad \forall i \in I, j \in J
\end{gathered}
$$

Equation (8) can then be rewritten as

$$
\sup _{P \in \mathbb{P}} P\left(\sum_{i \in I} M_{i j} \tilde{d}_{i} y_{i j} \leq U x_{j}\right) \geq 1-\varepsilon, \quad \forall j \in J
$$

Let $\mathrm{VAR}_{1-\varepsilon, P}$ be the $1-\varepsilon$ quantile under probability distribution $\mathrm{P}$.

$$
\operatorname{VAR}_{1-\varepsilon, P}\left(\sum_{i \in I} M_{i j} \tilde{d}_{i} y_{i j}\right)=\inf _{x \in \mathbb{R}}\left\{P\left(\sum_{i \in I} M_{i j} \tilde{d}_{i} y_{i j} \leq x\right) \geq 1-\varepsilon\right\}
$$

We know

$$
P\left(\sum_{i \in I} M_{i j} \tilde{d}_{i} y_{i j} \leq U x_{j}\right) \geq 1-\varepsilon \Rightarrow \operatorname{VAR}_{1-\varepsilon, P}\left(\sum_{i \in I} M_{i j} \tilde{d}_{i} y_{i j}\right) \leq U x_{j}
$$

Therefore equation (9) can be rewritten as

$$
\begin{gathered}
\sup _{P \in \mathbb{P}} \operatorname{VAR}_{1-\varepsilon, P}\left(\sum_{i \in I} M_{i j} \tilde{d}_{i} y_{i j}\right) \leq U x_{j} \\
\sup _{P \in \mathbb{P}} \operatorname{VAR}_{1-\varepsilon, P}\left(\sum_{i \in I_{j}} \tilde{d}_{i}\right) \leq U x_{j}
\end{gathered}
$$

Where $I_{j}=\left\{i \in I, y_{i j}=1\right\}$

If the following three conditions are satisfied

$$
\begin{aligned}
& P\left(\tilde{d}_{i} \in\left[\underline{d}_{i}, \bar{d}_{i}\right]\right)=1, \forall i \in I, j \in J, P \in \mathbb{P} \\
& \mathbb{E}_{P}\left[\tilde{d}_{i}\right]=d_{i}, \quad \forall i \in I, j \in J, P \in \mathbb{P} \\
& \mathbb{E}_{P}\left[\left(\tilde{d}_{i}-d_{i}\right)^{2}\right] \leq \sigma_{i}^{2}, \quad \forall i \in I, j \in J, P \in \mathbb{P}
\end{aligned}
$$

Based on theorem 2 and proposition 3 of (Ghosal and Wiesemann, 2020), for all probability distributions $P \in \mathbb{P}$ satisfying equations $(10,11,12)$, we have

$$
\sup _{P \in \mathbb{P}} \operatorname{VAR}_{1-\varepsilon, P}\left(\sum_{i \in I_{j}} \tilde{d}_{i}\right)=\sum_{i \in I_{j}} \sup _{P \in \mathbb{P}} \operatorname{VAR}_{1-\varepsilon, P}\left(\tilde{d}_{i}\right)
$$


and

$$
\sup _{P \in \mathbb{P}} \operatorname{VAR}_{1-\varepsilon, P}\left(\tilde{d}_{i}\right)=d_{i}+\min \left\{\bar{d}_{i}-d_{i}, \frac{1-\varepsilon}{\varepsilon}\left(d_{i}-\underline{d}_{i}\right), \sqrt{\frac{1-\varepsilon}{\varepsilon} * \sigma_{i}^{2}}\right\} \quad \forall i \in I
$$

Therefore, we can substitute equation (8) with equation (13) to obtain the distributionally robust formulation.

$$
\sum_{i \in I} M_{i j} d_{i}^{*} y_{i j} \leq U x_{j}, \quad \forall j \in J
$$

where,

$$
d_{i}^{*}=d_{i}+\min \left\{\bar{d}_{i}-d_{i}, \frac{1-\varepsilon}{\varepsilon}\left(d_{i}-\underline{d}_{i}\right), \sqrt{\frac{1-\varepsilon}{\varepsilon} * \sigma_{i}^{2}}\right\} \quad \forall i \in I
$$

$\bar{d}_{i}$ and $\underline{d}_{i}$ are the upper and lower bounds for $\tilde{d}_{i}$ determined by the deviation parameter, $\gamma(14-15)$. Further, to simplify considering a large variety of distributions, we utilize the Bhatia-Davis inequality (Bhatia and Davis, 2000) shown in (16). (16) provides upper bounds for variance for any bounded probability distribution. For numerical analysis, the solution will be tested assuming a uniform distribution such that the variance will be in accordance with a uniform distribution (17), which is easily verified to be within the bounds of (16).

$$
\begin{aligned}
\bar{d}_{i} & =(1+\gamma) d_{i} \\
\underline{d}_{i} & =(1-\gamma) d_{i} \\
\text { Bhatia-Davis inequality: } \quad \sigma_{i}^{2} & \leq\left(\bar{d}_{i}-d_{i}\right)\left(d_{i}-\underline{d}_{i}\right) \\
\sigma_{i}^{2} & =\frac{1}{3} \gamma^{2} d_{i}^{2}
\end{aligned}
$$

\subsection{Minimum Cost FLP}

The minimum cost facility location model utilizes a capacitated model presented by (Beasley, 1988). The objective of minimizing cost is more applicable to the private sector.

\subsubsection{Nomenclature}

Sets

I Set of demand points 
J Set of potential facility locations

Indices

$i \in I$

$j \in J$

Parameters

$d_{i} \quad$ Demand of point $i$

$f_{j} \quad$ Fixed cost for establishing facility at point $j$

$u_{j} \quad$ Capacity of facility of location $j$

$d_{i} \quad$ Cost of meeting demand at point $i$ from facility $j$

\section{Decision Variables}

$y_{j} \quad$ Binary: Equal to 1 if a facility is located at $j ; 0$ otherwise

$x_{i j} \quad$ Fractional: Equal to fraction of demand point $i$ met by facility is located at $j$

\subsubsection{Deterministic Formulation}

The objective (18) is to minimize the sum of the fixed and variable costs. (19) restricts the fractional demand variable, $x_{i j}$, to be equal to one across a set of facility locations. This implies that all demand must be met. (20) ensures demand does not exceed capacity. (21) restricts the fraction of demand to be allocated only if a facility is located. $(22,23)$ are variable definitions.

minimize $\quad \sum_{j \in J} f_{j} y_{j}+\sum_{i \in I} \sum_{j \in J} c_{i j} x_{i j}$

S.To.

$$
\begin{aligned}
& \sum_{j \in J} x_{i j}=1, \quad \forall i \in I \\
& \sum_{i \in I} d_{i} x_{i j} \leq u_{j} y_{j}, \quad \forall j \in J \\
& x_{i j} \leq y_{j}, \quad \forall i \in I, j \in J \\
& x_{i j} \in[0,1], \quad \forall i \in I, j \in J \\
& y_{j} \in\{0,1\}, \quad \forall j \in J
\end{aligned}
$$

\subsubsection{Robust Formulation}

Using the deterministic formulation, (24) is used in place of (20) to create a robust model. 
minimize $\quad \sum_{j \in J} f_{j} y_{j}+\sum_{i \in I} \sum_{j \in J} c_{i j} x_{i j}$

S.To.

$$
\begin{aligned}
& \sum_{j \in J} x_{i j}=1, \quad \forall i \in I \\
& P\left(\sum_{i \in I} \tilde{d}_{i} x_{i j} \leq u_{j} y_{j}\right) \leq 1-\varepsilon, \quad \forall j \in J, P \in \mathbb{P} \\
& x_{i j} \leq y_{j}, \quad \forall i \in I, j \in J \\
& x_{i j} \in[0,1], \quad \forall i \in I, j \in J \\
& y_{j} \in\{0,1\}, \quad \forall j \in J
\end{aligned}
$$

Equation (24) can then be rewritten as

$$
\sup _{P \in \mathbb{P}} P\left(\sum_{i \in I} \tilde{d}_{i} x_{i j} \leq u_{j} y_{j}\right) \geq 1-\varepsilon, \quad \forall j \in J
$$

Let $\mathrm{VAR}_{1-\varepsilon, P}$ be the $1-\varepsilon$ quantile under probability distribution $\mathrm{P}$.

$$
\operatorname{VAR}_{1-\varepsilon, P}\left(\sum_{i \in I} \tilde{d}_{i} x_{i j}\right)=\inf _{x \in \mathbb{R}}\left\{P\left(\sum_{i \in I} \tilde{d}_{i} x_{i j} \leq x\right) \geq 1-\varepsilon\right\}
$$

We know

$$
P\left(\sum_{i \in I} \tilde{d}_{i} x_{i j} \leq u_{j} y_{j}\right) \geq 1-\varepsilon \Rightarrow \operatorname{VAR}_{1-\varepsilon, P}\left(\tilde{d}_{i} x_{i j}\right) \leq u_{j} y_{j}
$$

Therefore equation (25) can be rewritten as

$$
\begin{aligned}
& \sup _{P \in \mathbb{P}} \operatorname{VAR}_{1-\varepsilon, P}\left(\sum_{i \in I} \tilde{d}_{i} x_{i j}\right) \leq u_{j} y_{j} \\
& \sup _{P \in \mathbb{P}} \operatorname{VAR}_{1-\varepsilon, P}\left(\sum_{i \in I_{j}} \tilde{d}_{i}\right) \leq u_{j} y_{j}
\end{aligned}
$$

Where $I_{j}=\left\{i \in I, x_{i j}=1\right\}$

If the following three conditions are satisfied:

$$
\begin{array}{r}
P\left(\tilde{d}_{i} \in\left[\underline{d}_{i}, \bar{d}_{i}\right]\right)=1, \quad \forall i \in I, j \in J, P \in \mathbb{P} \\
\mathbb{E}_{P}\left[\tilde{d}_{i}\right]=d_{i}, \quad \forall i \in I, j \in J, P \in \mathbb{P} \\
\mathbb{E}_{P}\left[\left(\tilde{d}_{i}-d_{i}\right)^{2}\right] \leq \sigma_{i}^{2}, \quad \forall i \in I, j \in J, P \in \mathbb{P}
\end{array}
$$


Based on theorem 2 and proposition 3 of (Ghosal and Wiesemann, 2020), for all probability distributions $P \in \mathbb{P}$ satisfying equations $(26,27,28)$, we have

$$
\sup _{P \in \mathbb{P}} \operatorname{VAR}_{1-\varepsilon, P}\left(\sum_{i \in I_{j}} \tilde{d}_{i}\right)=\sum_{i \in I_{j}} \sup _{P \in \mathbb{P}} \operatorname{VAR}_{1-\varepsilon, P}\left(\tilde{d}_{i}\right)
$$

and

$$
\sup _{P \in \mathbb{P}} \operatorname{VAR}_{1-\varepsilon, P}\left(\tilde{d}_{i}\right)=d_{i}+\min \left\{\bar{d}_{i}-d_{i}, \frac{1-\varepsilon}{\varepsilon}\left(d_{i}-\underline{d}_{i}\right), \sqrt{\frac{1-\varepsilon}{\varepsilon} * \sigma_{i}^{2}}\right\} \quad \forall i \in I
$$

Therefore, we can substitute equation (24) with equation (29) to obtain the distributionally robust formulation.

$$
\sum_{i \in I} d_{i}^{*} x_{i j} \leq u_{j} y_{j}, \quad \forall j \in J
$$

where,

$$
d_{i}^{*}=d_{i}+\min \left\{\bar{d}_{i}-d_{i}, \frac{1-\varepsilon}{\varepsilon}\left(d_{i}-\underline{d}_{i}\right), \sqrt{\frac{1-\varepsilon}{\varepsilon} * \sigma_{i}^{2}}\right\} \quad \forall i \in I
$$

Similar to the maximum coverage facility location problem, $\bar{d}_{i}$ and $\underline{d}_{i}$ are the upper and lower bounds for $\tilde{d}_{i}$ determined by the deviation parameter, $\gamma$ in accordance with equations (14-15). Inequality (16) shows how the variance is determined. 


\section{NUMERICAL ANALYSIS AND RESULTS}

For numerical analysis, simple Monte Carlo simulation (MCS) was used to test the feasibility of the models' solutions. When infeasibility was encountered, a greedy heuristic was employed to arrive at a feasible solution which would be used for evaluation.

\subsection{Maximum Coverage FLP}

The robust and deterministic models were solved using the network adopted from (Osman and Christofides, 1994) shown in table 1 . The robust model was always solved with the probability parameter $\varepsilon$ equal to 0.1 . Other input parameters will be varied and explored in later sections. 1000 MCS iterations were conducted with the models' solutions. Model coverage was calculated as the fraction of nominal demand covered over total nominal demand in the system. For each solution, different ranges were set on the demand. Using these bounds, uncertain demands were randomly generated in accordance with a uniform distribution for each MCS iteration. For each range of demands, different capacities or coverage radii were tested to display the behavior of the models' coverage. Infeasibility was then calculated by checking if deterministic constraint (2) or robust constraint (13) was satisfied for each iteration. When infeasibility was encountered, the greedy heuristic removed the smallest demand points until infeasibility was equal to zero. The greedy coverage shows this new coverage and coverage reduction shows how much coverage was removed after using the greedy heuristic.

\begin{tabular}{|c|c|c|c|}
\hline index i & x coordinate & y coordinate & demand \\
\hline 1 & 2 & 62 & 3 \\
\hline 2 & 80 & 25 & 14 \\
\hline 3 & 36 & 88 & 1 \\
\hline 4 & 57 & 23 & 14 \\
\hline 5 & 33 & 17 & 19 \\
\hline 6 & 76 & 43 & 2 \\
\hline 7 & 77 & 85 & 14 \\
\hline 8 & 94 & 6 & 6 \\
\hline 9 & 89 & 11 & 7 \\
\hline 10 & 59 & 72 & 6 \\
\hline 11 & 39 & 82 & 10 \\
\hline 12 & 87 & 24 & 18 \\
\hline 13 & 44 & 76 & 3 \\
\hline 14 & 2 & 83 & 6 \\
\hline 15 & 19 & 43 & 20 \\
\hline 16 & 5 & 27 & 4 \\
\hline 17 & 58 & 72 & 14 \\
\hline
\end{tabular}

\begin{tabular}{|c|c|c|c|}
\hline index i & x coordinate & y coordinate & demand \\
\hline 26 & 12 & 2 & 16 \\
\hline 27 & 53 & 33 & 3 \\
\hline 28 & 53 & 10 & 7 \\
\hline 29 & 33 & 32 & 14 \\
\hline 30 & 69 & 67 & 17 \\
\hline 31 & 43 & 5 & 3 \\
\hline 32 & 10 & 75 & 3 \\
\hline 33 & 8 & 26 & 12 \\
\hline 34 & 3 & 1 & 14 \\
\hline 35 & 96 & 22 & 20 \\
\hline 36 & 6 & 48 & 13 \\
\hline 37 & 59 & 22 & 10 \\
\hline 38 & 66 & 69 & 9 \\
\hline 39 & 22 & 50 & 6 \\
\hline 40 & 75 & 21 & 18 \\
\hline 41 & 4 & 81 & 7 \\
\hline 42 & 41 & 97 & 20 \\
\hline
\end{tabular}




\begin{tabular}{|c|c|c|c|}
18 & 14 & 50 & 11 \\
\hline 19 & 43 & 18 & 19 \\
\hline 20 & 87 & 7 & 15 \\
\hline 21 & 11 & 56 & 15 \\
\hline 22 & 31 & 16 & 4 \\
\hline 23 & 51 & 94 & 13 \\
\hline 24 & 55 & 13 & 13 \\
\hline 25 & 84 & 57 & 5 \\
\hline
\end{tabular}

\begin{tabular}{|c|c|c|c|}
43 & 92 & 34 & 9 \\
\hline 44 & 12 & 64 & 1 \\
\hline 45 & 60 & 84 & 8 \\
\hline 46 & 35 & 100 & 5 \\
\hline 47 & 38 & 2 & 1 \\
\hline 48 & 9 & 9 & 7 \\
\hline 49 & 54 & 59 & 9 \\
\hline 50 & 1 & 58 & 2 \\
\hline
\end{tabular}

Table 1: Network of Maximum Coverage FLP

\subsubsection{Effect of Varying Capacity}

For this analysis, an infinite coverage radius was assumed, and five facilities were located. Capacity was tested between 70 and 140 .

\begin{tabular}{|c|c|c|c|c|c|c|}
\hline Model & Capacity & $\begin{array}{l}\text { Demand } \\
\text { Range }\end{array}$ & \begin{tabular}{|l} 
Model \\
Coverage
\end{tabular} & Infeasibility & $\begin{array}{c}\text { Greedy } \\
\text { Coverage }\end{array}$ & $\begin{array}{l}\text { Coverage } \\
\text { Reduction }\end{array}$ \\
\hline \multirow{16}{*}{ Deterministic } & 70 & \multirow{8}{*}[0.8\mathrm{d},1.2\mathrm{d}]{} & 0.714 & 0.965 & 0.68 & 0.035 \\
\hline & 80 & & 0.816 & 0.982 & 0.784 & 0.032 \\
\hline & 90 & & 0.918 & 0.964 & 0.888 & 0.031 \\
\hline & 100 & & 1 & 0.924 & 0.981 & 0.019 \\
\hline & 110 & & 1 & 0.91 & 0.978 & 0.022 \\
\hline & 120 & & 1 & 0.917 & 0.977 & 0.023 \\
\hline & 130 & & 1 & 0.885 & 0.983 & 0.017 \\
\hline & 140 & & 1 & 0.886 & 0.981 & 0.019 \\
\hline & 70 & \multirow{8}{*}[0,2d]{} & 0.714 & 0.963 & 0.591 & 0.124 \\
\hline & 80 & & 0.816 & 0.975 & 0.683 & 0.133 \\
\hline & 90 & & 0.918 & 0.964 & 0.775 & 0.143 \\
\hline & 100 & & 1 & 0.957 & 0.868 & 0.132 \\
\hline & 110 & & 1 & 0.939 & 0.869 & 0.131 \\
\hline & 120 & & 1 & 0.934 & 0.863 & 0.137 \\
\hline & 130 & & 1 & 0.869 & 0.887 & 0.113 \\
\hline & 140 & & 1 & 0.879 & 0.883 & 0.117 \\
\hline \multirow{9}{*}{ Robust } & 70 & \multirow{8}{*}[0.8\mathrm{d},1.2\mathrm{d}]{} & 0.592 & 0 & 0.592 & 0 \\
\hline & 80 & & 0.673 & 0 & 0.673 & 0 \\
\hline & 90 & & 0.765 & 0 & 0.765 & 0 \\
\hline & 100 & & 0.847 & 0 & 0.847 & 0 \\
\hline & 110 & & 0.929 & 0 & 0.929 & 0 \\
\hline & 120 & & 1 & 0 & 1 & 0 \\
\hline & 130 & & 1 & 0 & 1 & 0 \\
\hline & 140 & & 1 & 0 & 1 & 0 \\
\hline & 70 & {$[0,2 d]$} & 0.357 & 0 & 0.357 & 0 \\
\hline
\end{tabular}




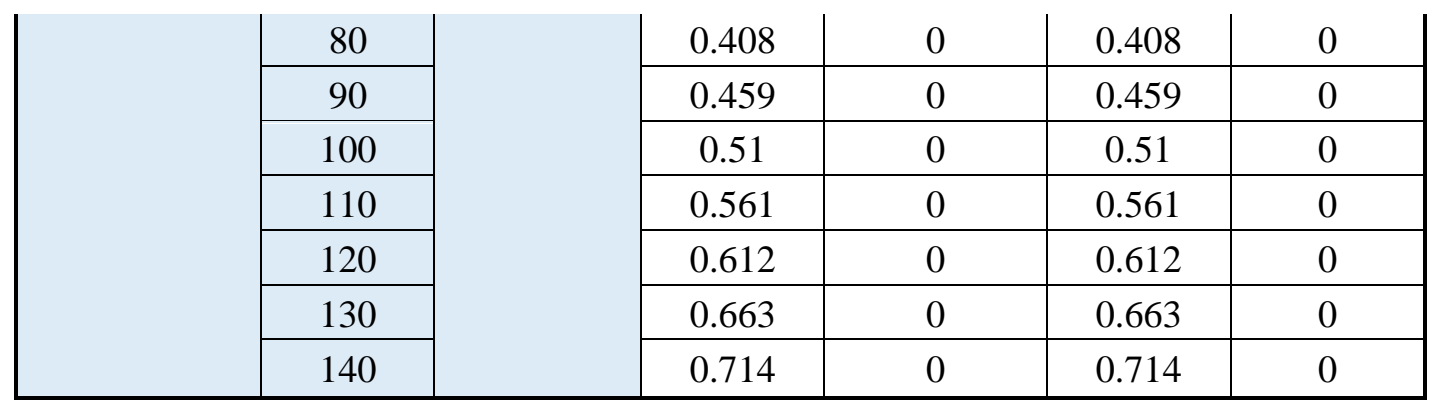

Table 2: Varying Capacity

Table 2 shows the effect of varying capacity in the models. For the deterministic, higher capacities resulted in higher model coverage, higher greedy coverage, lower infeasibility, and a lower coverage reduction. A larger deviation of demand results in higher infeasibilities for capacities higher than 90, and lower infeasibilities for capacities lower than 90. Because the infeasibility is often equal to 1 for higher capacities, varying the coverage radius will also be explored.

For the robust model, infeasibility is always equal to 0 . The greedy heuristic does not remove any demands and thus the greedy coverage is equal to the model coverage. Unlike the deterministic, the robust model coverage changes as distribution changes. In this analysis, for lower deviations, the robust model outperforms the deterministic model when coverage is greater than 120, and the deterministic outperforms the robust for capacities less than 120. For higher deviation, the deterministic model always provides better coverage than the robust model.

\subsubsection{Effect of Varying Coverage Radius}

For this experiment, 5 facilities are located, and the capacity is 122.5 . Coverage radius is varied ranging from 20 to 55 .

\begin{tabular}{|c|c|c|c|c|c|c|}
\hline Model & $\begin{array}{c}\text { Coverage } \\
\text { Radius }\end{array}$ & $\begin{array}{l}\text { Demand } \\
\text { Range }\end{array}$ & $\begin{array}{c}\text { Model } \\
\text { Coverage }\end{array}$ & Infeasibility & $\begin{array}{c}\text { Greedy } \\
\text { Coverage }\end{array}$ & $\begin{array}{l}\text { Coverage } \\
\text { Reduction }\end{array}$ \\
\hline \multirow{9}{*}{ Deterministic } & 20 & \multirow{8}{*}[0.8\mathrm{d},1.2\mathrm{d}]{} & 0.867 & 0 & 0.867 & 0 \\
\hline & 25 & & 0.961 & 0.049 & 0.961 & 0 \\
\hline & 30 & & 1 & 0.188 & 0.998 & 0.002 \\
\hline & 35 & & 1 & 0.365 & 0.997 & 0.003 \\
\hline & 40 & & 1 & 0.225 & 0.997 & 0.003 \\
\hline & 45 & & 1 & 0.568 & 0.99 & 0.01 \\
\hline & 50 & & 1 & 0.468 & 0.996 & 0.004 \\
\hline & 55 & & 1 & 0.614 & 0.993 & 0.007 \\
\hline & 20 & {$[0,2 \mathrm{~d}]$} & 0.867 & 0.452 & 0.843 & 0.024 \\
\hline
\end{tabular}




\begin{tabular}{|c|c|c|c|c|c|c|}
\hline & 25 & & 0.961 & 0.679 & 0.911 & 0.05 \\
\hline & 30 & & 1 & 0.728 & 0.941 & 0.059 \\
\hline & 35 & & 1 & 0.788 & 0.928 & 0.072 \\
\hline & 40 & & 1 & 0.829 & 0.919 & 0.081 \\
\hline & 45 & & 1 & 0.72 & 0.932 & 0.068 \\
\hline & 50 & & 1 & 0.707 & 0.952 & 0.048 \\
\hline & 55 & & 1 & 0.751 & 0.935 & 0.065 \\
\hline \multirow{6}{*}{ Robust } & 20 & \multirow{4}{*}[0.8\mathrm{d},1.2\mathrm{d}]{} & 0.845 & 0 & 0.845 & 0 \\
\hline & 25 & & 0.918 & 0 & 0.918 & 0 \\
\hline & 30 & & 0.973 & 0 & 0.973 & 0 \\
\hline & 35 & & 1 & 0 & 1 & 0 \\
\hline & 20 & \multirow{2}{*}[0,2\mathrm{d}]{} & 0.622 & 0 & 0.622 & 0 \\
\hline & 55 & & 0.622 & 0 & 0.622 & 0 \\
\hline
\end{tabular}

Table 3: Varying Coverage Radius

For the deterministic model there was more variation in the behavior of infeasibility, greedy coverage, and coverage reduction as the coverage radius increased shown in table 3 . This is expected because the demand points which are available to a facility alter as the coverage radius changes. In general, as the coverage radius increased, the infeasibility increased. The greedy coverage also increased until coverage radius was equal to 30 . For coverage radii greater than 30 , there was no significant change to greedy coverage or coverage reduction. A coverage radius of 30 produced the most optimal greedy coverage for both ranges of demand.

The robust model produced similar results as shown in section 5.1.1. The MCS shows no infeasibility, and the greedy heuristic does not change the coverage. Using a larger variation range of demands shows that coverage does not change as the coverage radius is increased from 20 to 55. For this analysis, the deterministic model always outperforms the robust model, expect when the variation range of demand is less and the coverage radius is at least 35 units.

\subsubsection{Effect of Varying Facilities Located}

For this experiment, the number of facilities located ranges from 1 to 8 , the capacity of each facility is determined in accordance using the approximation proposed by (Pirkul and Schilling, 1989), and an infinite coverage radius is considered.

\begin{tabular}{|c|c|c|c|c|c|c|c|}
\hline Model & Facilities & $\begin{array}{c}\text { Demand } \\
\text { Range }\end{array}$ & Capacity & $\begin{array}{c}\text { Model } \\
\text { Coverage }\end{array}$ & Infeasibility & $\begin{array}{c}\text { Greedy } \\
\text { Coverage }\end{array}$ & $\begin{array}{c}\text { Coverage } \\
\text { Reduction }\end{array}$ \\
\hline Deterministic & 1 & {$[0.8 \mathrm{~d}, 1.2 \mathrm{~d}]$} & 612.5 & 1 & 0 & 1 & 0 \\
\hline
\end{tabular}




\begin{tabular}{|c|c|c|c|c|c|c|c|}
\hline & 2 & & 306.25 & 1 & 0.474 & 0.993 & 0.007 \\
\hline & 3 & & 204.17 & 1 & 0.725 & 0.989 & 0.011 \\
\hline & 4 & & 153.13 & 1 & 0.844 & 0.984 & 0.016 \\
\hline & 5 & & 122.5 & 1 & 0.928 & 0.978 & 0.022 \\
\hline & 6 & & 102.08 & 1 & 0.941 & 0.978 & 0.022 \\
\hline & 7 & & 87.5 & 1 & 0.926 & 0.981 & 0.019 \\
\hline & 8 & & 76.56 & 1 & 0.941 & 0.974 & 0.026 \\
\hline & 1 & \multirow{8}{*}[0,2\mathrm{d}]{} & 612.5 & 1 & 0.004 & 1 & 0 \\
\hline & 2 & & 306.25 & 1 & 0.488 & 0.941 & 0.059 \\
\hline & 3 & & 204.17 & 1 & 0.739 & 0.904 & 0.096 \\
\hline & 4 & & 153.13 & 1 & 0.878 & 0.885 & 0.115 \\
\hline & 5 & & 122.5 & 1 & 0.938 & 0.862 & 0.138 \\
\hline & 6 & & 102.08 & 1 & 0.939 & 0.876 & 0.124 \\
\hline & 7 & & 87.5 & 1 & 0.958 & 0.873 & 0.127 \\
\hline & 8 & & 76.56 & 1 & 0.976 & 0.855 & 0.145 \\
\hline \multirow{16}{*}{ Robust } & 1 & \multirow{8}{*}[0.8\mathrm{d},1.2\mathrm{d}]{} & 612.5 & 1 & 0 & 1 & 0 \\
\hline & 2 & & 306.25 & 1 & 0 & 1 & 0 \\
\hline & 3 & & 204.17 & 1 & 0 & 1 & 0 \\
\hline & 4 & & 153.13 & 1 & 0 & 1 & 0 \\
\hline & 5 & & 122.5 & 1 & 0 & 1 & 0 \\
\hline & 6 & & 102.08 & 1 & 0 & 1 & 0 \\
\hline & 7 & & 87.5 & 1 & 0 & 1 & 0 \\
\hline & 8 & & 76.56 & 1 & 0 & 1 & 0 \\
\hline & 1 & & 612.5 & 0.624 & 0 & 0.624 & 0 \\
\hline & 2 & & 306.25 & 0.624 & 0 & 0.624 & 0 \\
\hline & 3 & & 204.17 & 0.624 & 0 & 0.624 & 0 \\
\hline & 4 & I & 153.13 & 0.620 & 0 & 0.620 & 0 \\
\hline & 5 & {$[0,2 \mathrm{~d}]$} & 122.5 & 0.622 & 0 & 0.622 & 0 \\
\hline & 6 & & 102.08 & 0.624 & 0 & 0.624 & 0 \\
\hline & 7 & & 87.5 & 0.614 & 0 & 0.614 & 0 \\
\hline & 8 & & 76.56 & 0.620 & 0 & 0.620 & 0 \\
\hline
\end{tabular}

Table 4: Varying Facilities Located

Using the capacity formula proposed by (Pirkul and Schilling, 1989) resulted in each model receiving $100 \%$ coverage when varying located facilities shown in table 4 . As the number of facilities increased in the deterministic model, infeasibility increased, greedy coverage decreased, and coverage reduction increased for both ranges. However, after 5 facilities were located, there was no significant change in the greedy heuristic's behavior. 
The robust model provides as much model coverage as the deterministic for small ranges of demand, and significantly less model coverage for large ranges of demand. For this analysis, the robust can outperform the deterministic by providing complete coverage with no infeasibility if small range distributions of demand can be expected. For large ranges of demand, the deterministic model is preferred.

\subsubsection{Comparing Symmetric and Various Asymmetric Distributions}

For this analysis, a coverage radius of 30,5 located facilities, and a capacity of 122.5 will be assumed.

\begin{tabular}{|c|c|c|c|c|c|c|}
\hline Model & Distribution & $\begin{array}{c}\text { Demand } \\
\text { Range }\end{array}$ & $\begin{array}{c}\text { Model } \\
\text { Coverage } \\
\end{array}$ & Infeasibility & $\begin{array}{c}\text { Greedy } \\
\text { Coverage }\end{array}$ & $\begin{array}{l}\text { Coverage } \\
\text { Reduction }\end{array}$ \\
\hline \multirow{8}{*}{ Deterministic } & \multirow{2}{*}{ symmetric } & {$[0.8 \mathrm{~d}, 1.2 \mathrm{~d}]$} & 1 & 0.197 & 0.998 & 0.002 \\
\hline & & {$[0,2 \mathrm{~d}]$} & 1 & 0.737 & 0.942 & 0.058 \\
\hline & \multirow{6}{*}{ Asymmetric } & {$[0.9 \mathrm{~d}, 1.2 \mathrm{~d}]$} & 1 & 0.73 & 0.991 & 0.009 \\
\hline & & {$[0.5 \mathrm{~d}, 2 \mathrm{~d}]$} & 1 & 0.998 & 0.857 & 0.143 \\
\hline & & {$[0.93 \mathrm{~d}, 1.2 \mathrm{~d}]$} & 1 & 0.91 & 0.985 & 0.015 \\
\hline & & {$[0.67 \mathrm{~d}, 2 \mathrm{~d}]$} & 1 & 1 & 0.817 & 0.183 \\
\hline & & {$[0.95 \mathrm{~d}, 1.2 \mathrm{~d}]$} & 1 & 0.971 & 0.982 & 0.018 \\
\hline & & {$[0.75 \mathrm{~d}, 2 \mathrm{~d}]$} & 1 & 1 & 0.801 & 0.199 \\
\hline \multirow{8}{*}{ Robust } & \multirow{2}{*}{ symmetric } & {$[0.8 \mathrm{~d}, 1.2 \mathrm{~d}]$} & 0.973 & 0 & 0.973 & 0 \\
\hline & & {$[0,2 \mathrm{~d}]$} & 0.622 & 0 & 0.622 & 0 \\
\hline & \multirow{6}{*}{ Asymmetric } & {$[0.9 \mathrm{~d}, 1.2 \mathrm{~d}]$} & 0.973 & 0 & 0.973 & 0 \\
\hline & & {$[0.5 \mathrm{~d}, 2 \mathrm{~d}]$} & 0.622 & 0 & 0.622 & 0 \\
\hline & & {$[0.93 \mathrm{~d}, 1.2 \mathrm{~d}]$} & 0.973 & 0 & 0.973 & 0 \\
\hline & & {$[0.67 \mathrm{~d}, 2 \mathrm{~d}]$} & 0.622 & 0 & 0.622 & 0 \\
\hline & & {$[0.95 \mathrm{~d}, 1.2 \mathrm{~d}]$} & 0.973 & 0 & 0.973 & 0 \\
\hline & & {$[0.75 \mathrm{~d}, 2 \mathrm{~d}]$} & 0.622 & 0 & 0.622 & 0 \\
\hline
\end{tabular}

Table 5: Symmetric vs Asymmetric Distributions

Table 5 summarizes the results comparing symmetric to asymmetric distributions. The deterministic model performs worse as the asymmetric distributions begin to favor demands higher than the nominal demand with greater probabilities. There is no effect from analyzing the robust model with a symmetric or an asymmetric distribution as the upper bound of the demand range controls the robust model. The better performing model is mostly important on the range of demands rather than the degree of asymmetry. Even when considering highly asymmetric demands to favor the robust model, the deterministic still performs better. 


\subsubsection{Maximum Coverage FLP Discussion}

The results in tables 2,3 , and 4 identify that when complete coverage cannot be achieved, the deterministic solution provides more coverage across a range of capacities, coverage radii, or located facilities, and various deviations of $\tilde{d}_{i}$. The capacity approximation to reach complete coverage proposed by (Pirkul and Schilling, 1989) is 122.5 when 5 facilities are located. It is not surprising that when capacity is above 120 for 5 located facilities, complete coverage is achieved, and the robust model performs better. Because the robust model is generated using the bounds of $\tilde{d}_{i}$, it provides a better mapping of variables compared with the deterministic. However, exploring different coverage radii and located facilities shows that even when a capacity of 122.5 is assumed, the deterministic model performs better until coverage radius equals 35 .

The comparison of symmetric to asymmetric distributions in table 5 showed how much better the deterministic is as providing coverage under a practical coverage radius of 30 . Even when considering very unlikely worst case asymmetric distributions, it is still observed that the deterministic performs better at providing maximum coverage. In practical application of FLPs, an infinite coverage radius would likely not be considered. However, there are other networks which could be explored with this formulation and there are scenarios in which coverage radius is not such an important factor.

\subsection{Minimum Cost FLP}

The network used in this model is adopted from (Beasley, 1988) and shown in tables 6, 7, and 8. The robust model was solved where the probability parameter equals 0.05. 10,000 MCS iterations were conducted with the models' solutions. Random demands were generated in accordance with symmetric, and asymmetric uniform distributions. Infeasibility was calculated by checking if deterministic constraint (20) or robust constraint (29) was satisfied for each iteration. After calculating infeasibility within the solution, the greedy heuristic was used to evaluate the cost of facilities which could meet demands.

\begin{tabular}{|c|c|c|c|c|c|c|c|c|c|c|}
\hline $\mathrm{i}$ & 1 & 2 & 3 & 4 & 5 & 6 & 7 & 8 & 9 & 10 \\
\hline demand & 146 & 87 & 672 & 1337 & 31 & 559 & 2370 & 1089 & 33 & 32 \\
\hline $\mathrm{i}$ & 11 & 12 & 13 & 14 & 15 & 16 & 17 & 18 & 19 & 20 \\
\hline demand & 5495 & 904 & 1466 & 143 & 615 & 564 & 226 & 3016 & 253 & 195 \\
\hline $\mathrm{i}$ & 21 & 22 & 23 & 24 & 25 & 26 & 27 & 28 & 29 & 30 \\
\hline
\end{tabular}




\begin{tabular}{|c|c|c|c|c|c|c|c|c|c|c|} 
demand & 38 & 807 & 551 & 304 & 814 & 337 & 4368 & 577 & 482 & 495 \\
\hline $\mathrm{i}$ & 31 & 32 & 33 & 34 & 35 & 36 & 37 & 38 & 39 & 40 \\
\hline demand & 231 & 322 & 685 & 12912 & 325 & 366 & 3671 & 2213 & 705 & 328 \\
\hline $\mathrm{i}$ & 41 & 42 & 43 & 44 & 45 & 46 & 47 & 48 & 49 & 50 \\
\hline demand & 1681 & 1117 & 275 & 500 & 2241 & 733 & 222 & 49 & 1464 & 222 \\
\hline
\end{tabular}

Table 6: Minimum Cost FLP Network (Demands)

\begin{tabular}{|c|c|c|c|c|c|c|c|c|c|c|c|c|c|c|c|c|}
\hline $\mathrm{j}$ & 1 & 2 & 3 & 4 & 5 & 6 & 7 & 8 & 9 & 10 & 11 & 12 & 13 & 14 & 15 & 16 \\
\hline capacity & 5000 & 5000 & 5000 & 5000 & 5000 & 5000 & 5000 & 5000 & 5000 & 5000 & 5000 & 5000 & 5000 & 5000 & 5000 & 5000 \\
\hline fixed cost & 7500 & 7500 & 7500 & 7500 & 7500 & 7500 & 7500 & 7500 & 7500 & 7500 & 0 & 7500 & 7500 & 7500 & 7500 & 7500 \\
\hline
\end{tabular}

Table 7: Minimum Cost FLP Network (Capacities and Fixed Costs)

\begin{tabular}{|c|c|c|c|c|c|c|c|c|}
\hline $\mathrm{i}$ & 1 & 2 & 3 & 4 & 5 & 6 & 7 & 8 \\
\hline 1 & 6739.73 & 10355.05 & 7650.40 & 5219.50 & 5776.13 & 6641.18 & 4374.53 & 3847.10 \\
\hline 2 & 3204.86 & 5457.08 & 3845.40 & 2396.85 & 2628.49 & 3220.09 & 1838.96 & 2266.35 \\
\hline 3 & 4914.00 & 26409.60 & 19622.40 & 13876.80 & 9147.60 & 14977.20 & 21848.40 & 35330.40 \\
\hline 4 & 32372.11 & 29982.23 & 21024.33 & 29681.40 & 21275.01 & 20071.71 & 64292.99 & 80186.58 \\
\hline 5 & 1715.46 & 2152.18 & 1577.90 & 1061.75 & 1250.46 & 1363.61 & 1524.04 & 955.58 \\
\hline 6 & 6421.51 & 23701.60 & 16197.03 & 10383.43 & 7483.61 & 12332.94 & 15840.66 & 27251.25 \\
\hline 7 & 81972.38 & 28499.25 & 43134.00 & 65767.50 & 58805.63 & 48555.38 & 138615.38 & 155294.25 \\
\hline 8 & 33391.46 & 26544.38 & 6370.65 & 16770.60 & 13571.66 & 8861.74 & 51550.54 & 57907.58 \\
\hline 9 & 2020.84 & 2480.78 & 1869.45 & 1324.95 & 1525.84 & 1646.29 & 1817.06 & 1211.93 \\
\hline 10 & 1459.60 & 1995.20 & 1402.40 & 869.60 & 1050.80 & 1181.20 & 1133.20 & 546.40 \\
\hline 11 & 141015.44 & 205925.13 & 104130.25 & 12638.50 & 46089.31 & 66146.06 & 198300.81 & 220212.13 \\
\hline 12 & 17684.50 & 32069.40 & 15322.80 & 8429.80 & 1231.70 & 9073.90 & 32781.30 & 41335.40 \\
\hline 13 & 38207.63 & 42477.35 & 15319.70 & 15832.80 & 11526.43 & 5185.98 & 62653.18 & 71210.95 \\
\hline 14 & 1953.74 & 5044.33 & 4089.80 & 3428.43 & 2289.79 & 3530.31 & 5553.76 & 8308.30 \\
\hline 15 & 17181.56 & 36054.38 & 25399.50 & 16297.50 & 15828.56 & 21148.31 & 7310.81 & 21709.50 \\
\hline 16 & 25640.85 & 35602.50 & 25154.40 & 15763.80 & 18421.65 & 21255.75 & 18478.05 & 8135.70 \\
\hline 17 & 7031.43 & 10492.05 & 6305.40 & 2542.50 & 3918.28 & 4743.18 & 6856.28 & 7119.00 \\
\hline 18 & 78453.70 & 92515.80 & 36644.40 & 27445.60 & 23562.50 & 23034.70 & 126332.70 & 141375.00 \\
\hline 19 & 9452.71 & 12441.28 & 7754.45 & 3542.00 & 5082.14 & 6005.59 & 10274.96 & 10214.88 \\
\hline 20 & 8597.06 & 14113.13 & 10500.75 & 7254.00 & 7875.56 & 9152.81 & 5467.31 & 6371.63 \\
\hline 21 & 1581.28 & 2030.15 & 1326.20 & 693.50 & 924.83 & 1063.53 & 1628.78 & 1619.75 \\
\hline 22 & 23170.99 & 48702.45 & 36072.90 & 26166.98 & 23493.79 & 30494.51 & 14919.41 & 33813.30 \\
\hline 23 & 12087.56 & 19877.33 & 9670.05 & 3801.90 & 2252.21 & 5847.49 & 19650.04 & 23844.53 \\
\hline 24 & 4883.00 & 12851.60 & 10822.40 & 8930.00 & 6798.20 & 9435.40 & 11943.40 & 18148.80 \\
\hline 25 & 24063.88 & 39682.50 & 24603.15 & 11050.05 & 13644.68 & 18976.38 & 20197.38 & 24684.55 \\
\hline 26 & 4124.04 & 12148.85 & 8180.68 & 5611.05 & 2952.96 & 5851.16 & 11613.86 & 17111.18 \\
\hline 27 & 281463.00 & 406770.00 & 325852.80 & 253234.80 & 264755.40 & 294457.80 & 211356.60 & 210756.00 \\
\hline 28 & 11056.76 & 22113.53 & 11424.60 & 5582.48 & 2430.61 & 7436.09 & 19279.01 & 25460.13 \\
\hline 29 & 8585.63 & 22449.15 & 14122.60 & 7458.95 & 6609.43 & 10790.78 & 11525.83 & 20448.85 \\
\hline 30 & 12480.19 & 25455.38 & 22151.25 & 19069.88 & 15598.69 & 19892.81 & 23976.56 & 34080.75 \\
\hline 31 & 3727.76 & 11116.88 & 8229.38 & 5826.98 & 4628.66 & 6632.59 & 6476.66 & 11884.95 \\
\hline 32 & 4673.03 & 13346.90 & 7880.95 & 4330.90 & 2861.78 & 5655.13 & 9623.78 & 14610.75 \\
\hline 33 & 13451.69 & 35106.25 & 25927.25 & 17347.63 & 15249.81 & 21192.19 & 16808.19 & 32845.75 \\
\hline 34 & 372672.60 & 229188.00 & 203364.00 & 322800.00 & 261306.60 & 229995.00 & 681269.40 & 810550.80 \\
\hline 35 & 9745.94 & 18070.00 & 12049.38 & 7198.75 & 7592.81 & 9802.81 & 5780.94 & 10692.50 \\
\hline 36 & 12055.13 & 18181.05 & 11400.90 & 5307.00 & 7379.48 & 8870.93 & 10865.63 & 10202.25 \\
\hline 37 & 97602.71 & 73603.55 & 59561.98 & 83331.70 & 65940.34 & 56946.39 & 185247.84 & 222003.73 \\
\hline 38 & 60774.51 & 63568.43 & 27330.55 & 30982.00 & 20497.91 & 15076.06 & 97731.61 & 114578.08 \\
\hline 39 & 54470.06 & 65177.25 & 52117.13 & 40378.88 & 44494.31 & 47243.81 & 47631.56 & 34351.13 \\
\hline 40 & 7146.30 & 8618.20 & 6428.80 & 7822.80 & 5211.10 & 5621.10 & 15256.10 & 19762.00 \\
\hline 41 & 38011.61 & 70728.08 & 39587.55 & 15801.40 & 16494.81 & 27925.61 & 49862.66 & 62659.28 \\
\hline 42 & 39723.31 & 52917.88 & 32225.45 & 13627.40 & 20427.14 & 24504.19 & 41119.56 & 40854.28 \\
\hline 43 & 16111.56 & 20714.38 & 15620.00 & 11041.25 & 12598.44 & 13719.06 & 12502.19 & 7885.63 \\
\hline 44 & 16981.25 & 32575.00 & 23312.50 & 16250.00 & 16268.75 & 19856.25 & 9331.25 & 20750.00 \\
\hline 45 & 168663.26 & 210766.05 & 169251.53 & 131938.88 & 144628.54 & 153760.61 & 134768.14 & 109304.78 \\
\hline 46 & 57109.86 & 66703.00 & 53124.18 & 40919.73 & 45381.86 & 48057.31 & 52583.59 & 39050.58 \\
\hline
\end{tabular}




\begin{tabular}{|c|c|c|c|c|c|c|c|c|}
\hline 47 & 15576.08 & 18481.50 & 14368.95 & 10672.65 & 12024.08 & 12834.38 & 14205.23 & 10134.30 \\
\hline 48 & 2542.49 & 3928.58 & 3020.85 & 2205.00 & 2361.19 & 2682.14 & 1756.04 & 1983.28 \\
\hline 49 & 34056.30 & 34221.00 & 24448.80 & 31329.60 & 21905.10 & 20807.10 & 69009.30 & 86632.20 \\
\hline 50 & 7095.68 & 11999.10 & 7886.55 & 4190.25 & 4847.93 & 6351.98 & 4903.43 & 6421.35 \\
\hline $\mathrm{i}$ & 9 & 10 & 11 & 12 & 13 & 14 & 15 & 16 \\
\hline 1 & 6429.48 & 5396.53 & 5219.50 & 4182.90 & 7391.25 & 5038.83 & 10349.58 & 6051.70 \\
\hline 2 & 3117.86 & 2582.81 & 2296.80 & 1779.15 & 5115.60 & 2189.14 & 5399.44 & 2838.38 \\
\hline 3 & 15111.60 & 23679.60 & 9828.00 & 19303.20 & 57472.80 & 11180.40 & 22957.20 & 15489.60 \\
\hline 4 & 25921.09 & 69206.46 & 23096.68 & 48700.23 & 135170.70 & 40527.81 & 60515.96 & 52911.78 \\
\hline 5 & 1318.66 & 1789.09 & 1133.05 & 1015.25 & 2005.70 & 1379.89 & 2512.94 & 1823.58 \\
\hline 6 & 12444.74 & 17769.21 & 7029.43 & 13919.10 & 45474.65 & 6966.54 & 20326.64 & 10956.40 \\
\hline 7 & 53176.88 & 147325.13 & 56998.50 & 102384.00 & 259515.00 & 96429.38 & 131920.13 & 118381.50 \\
\hline 8 & 10985.29 & 57376.69 & 12741.30 & 33595.65 & 105796.35 & 32220.79 & 60071.96 & 46527.53 \\
\hline 9 & 1593.49 & 2099.21 & 1395.90 & 1275.45 & 1940.40 & 1663.61 & 2869.76 & 2135.93 \\
\hline 10 & 1134.80 & 1406.80 & 928.80 & 768.80 & 1950.40 & 1145.20 & 2314.80 & 1500.80 \\
\hline 11 & 58178.31 & 241573.94 & 25277.00 & 97536.25 & 461992.13 & 106122.19 & 288418.81 & 185456.25 \\
\hline 12 & 9254.70 & 37595.10 & 2463.40 & 19775.00 & 79235.60 & 16712.70 & 41956.90 & 28589.00 \\
\hline 13 & 11563.08 & 70496.28 & 10371.95 & 38482.50 & 135275.15 & 36631.68 & 77569.73 & 55891.25 \\
\hline 14 & 3558.91 & 5947.01 & 2434.58 & 4897.75 & 13134.55 & 3283.64 & 4634.99 & 4204.20 \\
\hline 15 & 20779.31 & 12569.06 & 14621.63 & 11931.00 & 39913.50 & 11169.94 & 32479.69 & 14375.63 \\
\hline 16 & 20437.95 & 23300.25 & 16271.40 & 12619.50 & 35094.90 & 19253.55 & 39867.75 & 24957.00 \\
\hline 17 & 4415.48 & 8788.58 & 3062.30 & 2712.00 & 17458.50 & 5031.33 & 13093.88 & 8294.20 \\
\hline 18 & 11423.10 & 144881.10 & 22846.20 & 74042.80 & 274079.00 & 75211.50 & 159433.30 & 114834.20 \\
\hline 19 & 5638.74 & 12438.11 & 4123.90 & 5635.58 & 21789.63 & 8073.86 & 16239.44 & 11726.55 \\
\hline 20 & 8870.06 & 6729.94 & 7312.50 & 5869.50 & 9506.25 & 6695.81 & 13554.94 & 7707.38 \\
\hline 21 & 1008.43 & 1953.68 & 780.90 & 931.95 & 3066.60 & 1298.18 & 2600.63 & 1846.80 \\
\hline 22 & 30655.91 & 11166.86 & 22333.73 & 20982.00 & 56025.98 & 17380.76 & 40178.51 & 15978.60 \\
\hline 23 & 6260.74 & 22873.39 & 0.00 & 10703.18 & 46945.20 & 10145.29 & 26881.91 & 17728.43 \\
\hline 24 & 9496.20 & 11616.60 & 7106.00 & 10898.40 & 28226.40 & 7117.40 & 7725.40 & 7911.60 \\
\hline 25 & 17796.08 & 27157.08 & 10541.30 & 5270.65 & 59259.20 & 14845.33 & 44597.03 & 26495.70 \\
\hline 26 & 5918.56 & 12852.34 & 3268.90 & 9073.73 & 29479.08 & 5623.69 & 13172.49 & 8745.15 \\
\hline 27 & 289434.60 & 239639.40 & 248102.40 & 222222.00 & 124051.20 & 238875.00 & 392519.40 & 261534.00 \\
\hline 28 & 7551.49 & 22351.54 & 1601.18 & 11698.68 & 49650.85 & 9022.84 & 26549.21 & 16963.80 \\
\hline 29 & 10887.18 & 14092.48 & 5916.55 & 8953.15 & 37089.90 & 2958.28 & 20575.38 & 9917.15 \\
\hline 30 & 19991.81 & 23444.44 & 16099.88 & 22275.00 & 50490.00 & 16118.44 & 8049.94 & 17411.63 \\
\hline 31 & 6678.79 & 6101.29 & 4440.98 & 6843.38 & 18722.55 & 3693.11 & 8596.09 & 3285.98 \\
\hline 32 & 5719.53 & 11338.43 & 2398.90 & 6931.05 & 26701.85 & 3900.23 & 13310.68 & 7961.45 \\
\hline 33 & 21329.19 & 15695.06 & 14693.25 & 19385.50 & 53121.75 & 10951.44 & 27888.06 & 7346.63 \\
\hline 34 & 258078.60 & 728398.20 & 261790.80 & 512606.40 & 1361570.4 & 451435.80 & 644793.00 & 575875.20 \\
\hline 35 & 9607.81 & 8559.69 & 6353.75 & 4891.25 & 21336.25 & 5951.56 & 17830.31 & 9514.38 \\
\hline 36 & 8340.23 & 13994.93 & 5984.10 & 4154.10 & 26946.75 & 8459.18 & 21470.48 & 13697.55 \\
\hline 37 & 73007.01 & 198738.76 & 65986.23 & 137295.40 & 378755.43 & 119995.81 & 174969.04 & 155375.08 \\
\hline 38 & 24757.94 & 109515.84 & 20525.58 & 63513.10 & 209073.18 & 58395.54 & 114439.76 & 87468.83 \\
\hline 39 & 46820.81 & 53659.31 & 42775.88 & 39250.88 & 40960.50 & 48318.94 & 73399.31 & 55712.63 \\
\hline 40 & 7047.90 & 16305.70 & 5658.00 & 11939.20 & 32644.20 & 9417.70 & 13583.30 & 12308.20 \\
\hline 41 & 26917.01 & 61965.86 & 10086.00 & 22567.43 & 133135.20 & 23134.76 & 81255.34 & 47404.20 \\
\hline 42 & 22884.54 & 50669.91 & 16196.50 & 20636.58 & 91957.03 & 31401.66 & 69686.84 & 47528.35 \\
\hline 43 & 13320.31 & 14853.44 & 11550.00 & 10071.88 & 13193.13 & 13052.19 & 23103.44 & 15654.38 \\
\hline 44 & 19556.25 & 9543.75 & 14550.00 & 13087.50 & 32087.50 & 12481.25 & 27518.75 & 12525.00 \\
\hline 45 & 150511.16 & 149278.61 & 136084.73 & 124879.73 & 89976.15 & 142499.59 & 224408.14 & 160511.63 \\
\hline 46 & 46994.46 & 58814.09 & 42605.63 & 40553.23 & 45922.45 & 49175.14 & 75966.29 & 61003.93 \\
\hline 47 & 12512.48 & 16103.33 & 11183.25 & 10561.65 & 14940.60 & 13172.93 & 21287.03 & 16755.45 \\
\hline 48 & 2611.09 & 2073.31 & 2174.38 & 1857.10 & 2006.55 & 2064.74 & 3788.31 & 2318.93 \\
\hline 49 & 27212.10 & 74352.90 & 23899.80 & 51935.40 & 147937.20 & 42986.70 & 64873.50 & 56510.40 \\
\hline 50 & 6030.08 & 6801.53 & 4001.55 & 2614.05 & 14979.45 & 4503.83 & 12617.93 & 7448.10 \\
\hline
\end{tabular}

Table 8: Minimum Cost FLP Network (Variable Costs) 


\subsubsection{Model Solution Networks}

Plots displaying which customers are mapped to which facility are generated to visualize each model's solution. These are shown in figures 1,2 , and 3 .

$$
\begin{gathered}
\bullet=\text { Customer } \\
\bullet=\text { Facility } \\
\gamma=\text { Facility Serving Customer }
\end{gathered}
$$

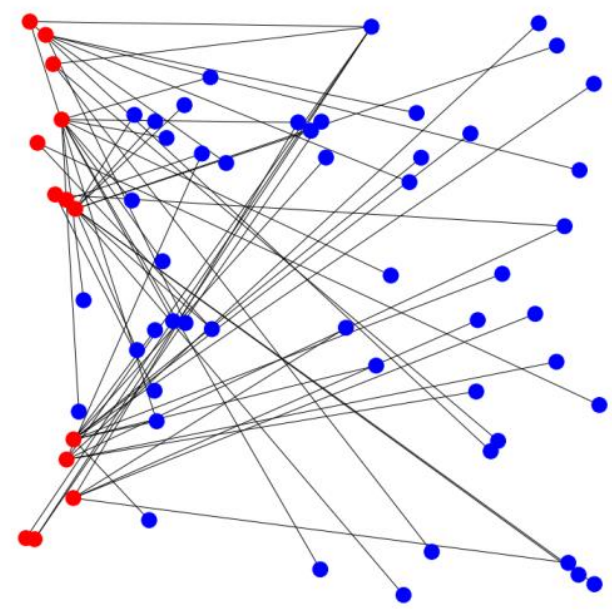

Figure 1: Deterministic Solution

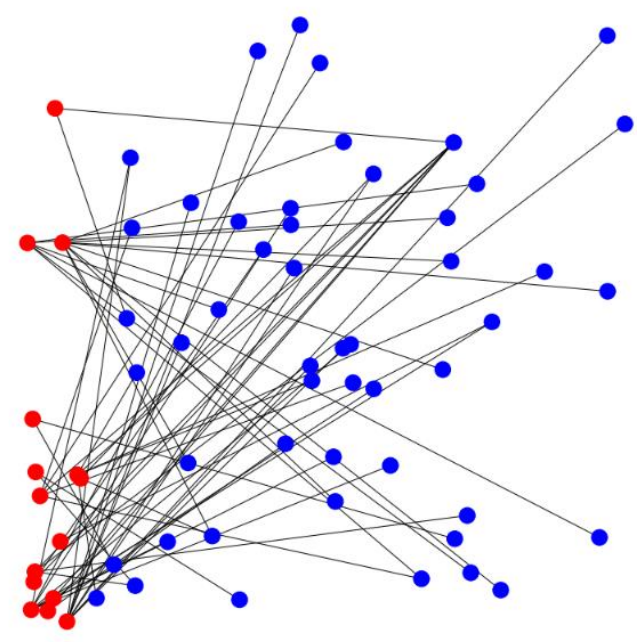

Figure 2: Robust Solution with $\gamma=0.2$

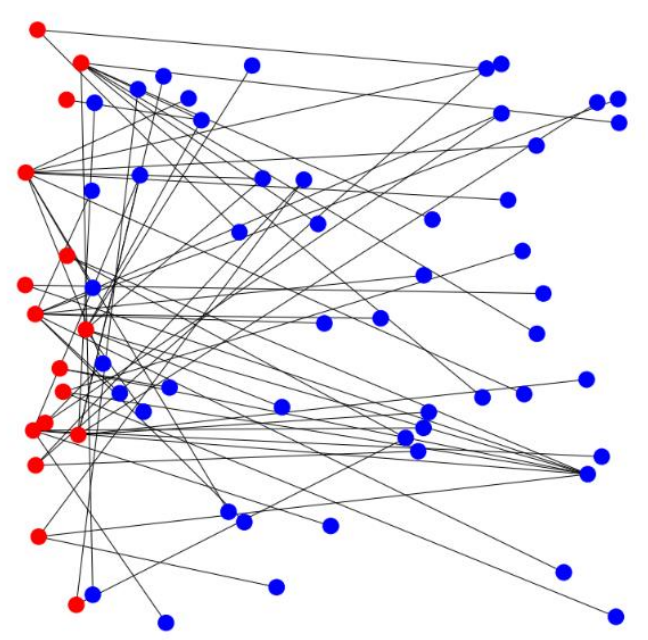

Figure 3 Robust Solution with $\gamma=0.35$

The mapping is clearly different in each solution. Changing the deviation on demands greatly alters how many facilities will be located and which facility will serve a customer. The deterministic solution locates the least facilities, and the robust solution locates more facilities 
when given greater deviations. For this network of 16 potential facilities, the deterministic model located 13 , the robust model with $\gamma=0.2$ located 15 , and the robust model with $\gamma=0.35$ located 16.

\subsubsection{MCS Results Considering Symmetric and Asymmetric Distributions}

The robust and deterministic models were solved using the input network and parameters outlined in section 5.2.

\begin{tabular}{|c|c|c|c|c|c|c|}
\hline Model & Distribution & $\begin{array}{c}\text { Demand } \\
\text { Range }\end{array}$ & $\begin{array}{c}\text { Objective } \\
\text { Value }\end{array}$ & Infeasibility & Greedy Cost & $\begin{array}{c}\text { Change in } \\
\text { Cost }\end{array}$ \\
\hline \multirow{4}{*}{ Deterministic } & \multirow{2}{*}{ Symmetric } & {$[0.8 \mathrm{~d}, 1.2 \mathrm{~d}]$} & $\$ 1,040,444.38$ & 0.9943 & $\$ 925,048.35$ & $-\$ 115,396.02$ \\
\hline & & {$[0.65 \mathrm{~d}, 1.35 \mathrm{~d}]$} & $\$ 1,040,444.38$ & 0.9940 & $\$ 898,010.44$ & $-\$ 142,433.93$ \\
\hline & \multirow{2}{*}{ Asymmetric } & {$[0.9 \mathrm{~d}, 1.2 \mathrm{~d}]$} & $\$ 1,040,444.38$ & 1 & $\$ 877,468.04$ & $-\$ 162,976.33$ \\
\hline & & {$[0.825 \mathrm{~d}, 1.35 \mathrm{~d}]$} & $\$ 1,040,444.38$ & 1 & $\$ 840,784.51$ & $-\$ 199,659.87$ \\
\hline \multirow{4}{*}{ Robust } & \multirow{2}{*}{ Symmetric } & {$[0.8 \mathrm{~d}, 1.2 \mathrm{~d}]$} & $\$ 1,183,964.33$ & 0 & $\$ 1,183,964.33$ & $\$ 0.00$ \\
\hline & & {$[0.65 \mathrm{~d}, 1.35 \mathrm{~d}]$} & $\$ 1,336,767.35$ & 0 & $\$ 1,336,767.35$ & $\$ 0.00$ \\
\hline & \multirow{2}{*}{ Asymmetric } & {$[0.9 \mathrm{~d}, 1.2 \mathrm{~d}]$} & $\$ 1,183,964.33$ & 0 & $\$ 1,183,964.33$ & $\$ 0.00$ \\
\hline & & {$[0.825 \mathrm{~d}, 1.35 \mathrm{~d}]$} & $\$ 1,336,767.35$ & 0 & $\$ 1,336,767.35$ & $\$ 0.00$ \\
\hline
\end{tabular}

Table 9: Minimum Cost FLP Results

Looking at the results from table 9, the robust solution consistently costed more, meaning that the deterministic model was more effective at minimizing the total cost of the network.

Accounting for infeasibility through the greedy heuristic shows that the deterministic cost is even less. However, this does not mean that the deterministic case is more effective. If this model were to be applied, the change in cost shows that more than $10 \%$ of a decision maker's investment will not meet consumer demands under the best-case symmetric distribution and small range and almost $20 \%$ of investments will not meet consumer demands under a more worse case asymmetric distribution and large range. The deterministic case may provide a less efficient solution.

\subsubsection{Considering Penalty}

To account for the effect of investment not meeting demand, we consider using a penalty parameter. This is a simple parameter which accounts for many factors including but not limited to the missed opportunity cost and the negative consumer perception caused from not meeting demands. The calculation for penalty is shown in (29). 


$$
\sum_{j \in J} f y_{j}+\sum_{i \in I} \sum_{j \in J} c_{i j} \tilde{x}_{i j}+\sum_{i \in I} \sum_{j \in J} \rho \tilde{d}_{i}\left(x_{i j}-\tilde{x}_{i j}\right)
$$

where,

$$
\begin{aligned}
& \rho=\text { penalty } \\
& \tilde{x}_{i j}=x_{i j} \text { after the greedy heuristic }
\end{aligned}
$$

\begin{tabular}{|c|c|c|c|}
\hline Demand Range & Penalty & $\begin{array}{c}\text { Cost after greedy } \\
\text { heuristic }\end{array}$ & Change in Cost \\
\hline$[0.8 \mathrm{~d}, 1.2 \mathrm{~d}]$ & 0 & $\$ 925,048.35$ & $-\$ 115,396.02$ \\
\hline$[0.65 \mathrm{~d}, 1.35 \mathrm{~d}]$ & 0 & $\$ 898,010.44$ & $-\$ 142,433.93$ \\
\hline$[0.8 \mathrm{~d}, 1.2 \mathrm{~d}]$ & 25 & $\$ 1,158,736.63$ & $\$ 118,292.25$ \\
\hline$[0.65 \mathrm{~d}, 1.35 \mathrm{~d}]$ & 25 & $\$ 1,165,224.90$ & $\$ 124,780.53$ \\
\hline$[0.8 \mathrm{~d}, 1.2 \mathrm{~d}]$ & 50 & $\$ 1,393,468.75$ & $\$ 353,024.37$ \\
\hline$[0.65 \mathrm{~d}, 1.35 \mathrm{~d}]$ & 50 & $\$ 1,425,226.54$ & $\$ 384,728.16$ \\
\hline
\end{tabular}

Table 10: Effect of Considering Penalty

Table 10 shows the effects of various penalties on the deterministic model's performance. The true value of penalty is unknown and varies based on location, products, market, and consumers. Because of this, a range of penalties are evaluated to capture these fluctuations. For penalties greater than 25 , the robust model outperforms the deterministic.

\subsubsection{Minimum Cost FLP Discussion}

The results in table 7 show that the deterministic solution provides a lower cost. However, it is not realistic to consider this lower cost as better with $99.4 \%$ infeasibility in the model. We are almost completely certain that some demands will not be covered. For a symmetric, uniform deviation of 0.2 , uncovered demands make up $11.1 \%$ of the objective cost and for a symmetric, uniform deviation of 0.35 , uncovered demands make up $13.7 \%$ of the objective cost. To rebalance the results, a penalty is added to account for shortages and lost opportunity cost. The penalty can vary based on local demands and prices, so table 10 shows results considering a range of values for penalty. It becomes clear that the penalty from not meeting demands can easily cause the robust model to outperform the deterministic. 


\subsection{Evaluation of $d_{i}^{*}$}

In the maximum coverage FLP, constraint (8) is satisfied with a probability of 1 . The reason that a lower confidence was not produced is because of how $d_{i}^{*}$ is evaluated from the second term in equation (13). For the demands and probability parameter used, the first entry of the minimum statement in (13) always controls. The result is that $d_{i}^{*}$ is equal to $\bar{d}_{i}$ for the demands used in this problem. Similarly, in the minimum cost FLP, constraint (24) is satisfied with a probability of 1 , and thus $d_{i}^{*}$ is equal to $\bar{d}_{i}$.

This result may be too conservative. While having $d_{i}^{*}$ equal to $\bar{d}_{i}$ does satisfy the constraints of (8) and (24), the initial goal was to have a parameter which could provide an upper limit for $\tilde{d}_{i}$ without using the upper bound. Doing so would cause a small amount infeasibility in the MCS and ultimately a more optimal solution. 


\section{CONCLUSION}

FLPs are network problems in which facilities are located to optimally meet objectives while satisfying constraints. Objectives and constraints vary based on the model's application to meet certain goals. The objective of minimizing cost is used in the private sector because reducing cost is directly correlated to maximizing asset utilization. Public sector applications include postal services, waste management, fire departments, police, and others. Maximizing coverage is a better objective to suit these applications because they provide essential services. For any FLP, the constraints are modeled to provide more control of the objective.

The two models considered in this paper, the minimum cost FLP and maximum coverage FLP, are archetypal examples of problems which have been popular in private or public sector applications. The constraints for the minimum cost FLP suit the idea of maximizing asset utilization because demands can be fractionally covered. The constraints for the maximum coverage FLP include a coverage radius, number of facilities to locate, and a binary decision variable to cover demands. These constraints also better fit the objective by giving more control over how coverage can be maximized.

Even with this effort, these models are both deterministic, and their solutions can only be accurate with accurate assumptions. However, parameters like demand, capacity, and cost are never exact. Natural market fluctuations and extreme events will always cause these parameters to deviate. To get a better picture of each problem, robust problems can be developed for both the maximum coverage FLP and minimum cost FLP by considering each demand as uncertain.

The methods by which the robust FLPs were developed in this paper provided convenient and fast computations. The deterministic maximum covering FLP provided better coverage when considering more common input parameters. However, there exist scenarios where the robust model performs better than the deterministic. The minimum cost FLP initially shows that the deterministic performs better than the robust. However, the deterministic solution was highly infeasible and penalty considerations show that the robust can easily outperform the deterministic.

For both problems and networks, the equivalent uncertain demand $d_{i}^{*}$ used in formulation was equal to the upper bound set by deviation. Other networks and problems should be explored to find scenarios where $d_{i}^{*}$ is not always equal to the upper bound. 


\section{REFERENCES}

1. "Code of Ethics." American Society of Civil Engineers (ASCE), www.asce.org/code-of-ethics/.

2. Church, R., and ReVell, C. (1974). "The maximal covering facility location problem." Papers of the Regional Science Association, 32(1), 101-118.

3. Current, J., Daskin, M., and Schilling, D. (2002). "Discrete network location models." Facility location: Applications and theory, 1, 81-118.

4. Esnaf, Ş., and Küçükdeniz, T. (2009). "A fuzzy clustering-based hybrid method for a multi-facility location problem.” Journal of Intelligent Manufacturing, 20(2), 259-265.

5. Arabani, A. B., and Farahani, R. Z. (2012). "Facility location dynamics: An overview of classifications and applications." Computers \& Industrial Engineering, 62(1), 408-420.

6. Chauhan, D., Unnikrishnan, A., and Figliozzi, M. (2019). "Maximum coverage capacitated facility location problem with range constrained drones." Transportation Research Part C: Emerging Technologies, 99, 1-18.

7. Karatas, M., and Dasci, A. (2020). "A two-level facility location and sizing problem for maximal coverage.” Computers \& Industrial Engineering, 139, 106204.

8. Arslan, O. (2021). "The location-or-routing problem." Transportation Research Part B: Methodological, 147, 1-21.

9. Snyder, L. (2006). "Facility location under uncertainty: a review." IIE transactions, 38(7), 547564.

10. Snyder, L. V., and Daskin, M. S. (2005). "Reliability models for facility location: the expected failure cost case.” Transportation Science, 39(3), 400-416.

11. Bertsimas, D., and Sim, M. (2004). "The price of robustness." Operations research, 52(1), 35-53.

12. Wang, Q., Batta, R., and Rump, C. M. (2002). "Algorithms for a facility location problem with stochastic customer demand and immobile servers." Annals of operations Research, 111(1), 1734.

13. Miranda, P. A., and Garrido, R. A. (2004). "Incorporating inventory control decisions into a strategic distribution network design model with stochastic demand." Transportation Research Part E: Logistics and Transportation Review, 40(3), 183-207.

14. Baron, O., Milner, J., and Naseraldin, H. (2011). "Facility location: A robust optimization approach." Production and Operations Management, 20(5), 772-785.

15. Gülpınar, N., Pachamanova, D., and Çanakoğlu, E. (2013). "Robust strategies for facility location under uncertainty." European Journal of Operational Research, 225(1), 21-35.

16. Naoum-Sawaya, J., and S. Elhedhli, S. (2013). "A stochastic optimization model for real-time ambulance redeployment." Computers \& Operations Research, 40(8), 1972-1978. 
17. Berglund, P. G., and Kwon, C. (2014). "Robust facility location problem for hazardous waste transportation." Networks and spatial Economics, 14(1), 91-116.

18. Lutter, P., Degel, D., Büsing, C., Koster, A., and Werners B., (2017). "Improved handling of uncertainty and robustness in set covering problems." European Journal of Operational Research, 263(1), 35-49.

19. Zhong, S., Cheng, R., Jiang, Y., Wang, Z., Larsen, A., and Nielsen, O. A. (2020). "Risk-averse optimization of disaster relief facility location and vehicle routing under stochastic demand." Transportation Research Part E: Logistics and Transportation Review, 141, 102015.

20. Chauhan, D. R., Unnikrishnan, A., Figliozzi, M., and Boyles, S. D. (2020). "Robust maximum coverage facility location problem with drones considering uncertainties in battery availability and consumption." Transportation Research Record, 2675(2) 25-39.

21. Basciftci, B., Ahmed, S., and Shen, S. (2021). Distributionally robust facility location problem under decision-dependent stochastic demand. European Journal of Operational Research, 292(2), 548-561.

22. Ghosal, S., and Wiesemann, W. (2020). "The distributionally robust chance constrained vehicle routing problem." Operations Research, 68(3), 716-732.

23. Bhatia, R., and Davis, C., (2000) "A better bound on the variance." The American Mathematical Monthly, 107(4):353-357.

24. Beasley, J., (1988). "An algorithm for solving large capacitated warehouse location problems." European Journal of Operational Research, 33, 314-325.

25. Osman, I.H., and Christofides, N. (1994). "Capacitated clustering problems by hybrid simulated annealing and tabu search." International Transactions in Operational Research, 1(3): 317-336.

26. Pirkul, H., and Schilling, D. (1989). "The capacitated maximal covering location problem with backup service." Annals of Operations Research, 18(1), 141-154. 


\section{APPENDIX}

\subsection{Python code for Maximum Coverage FLP}

\subsubsection{Deterministic}

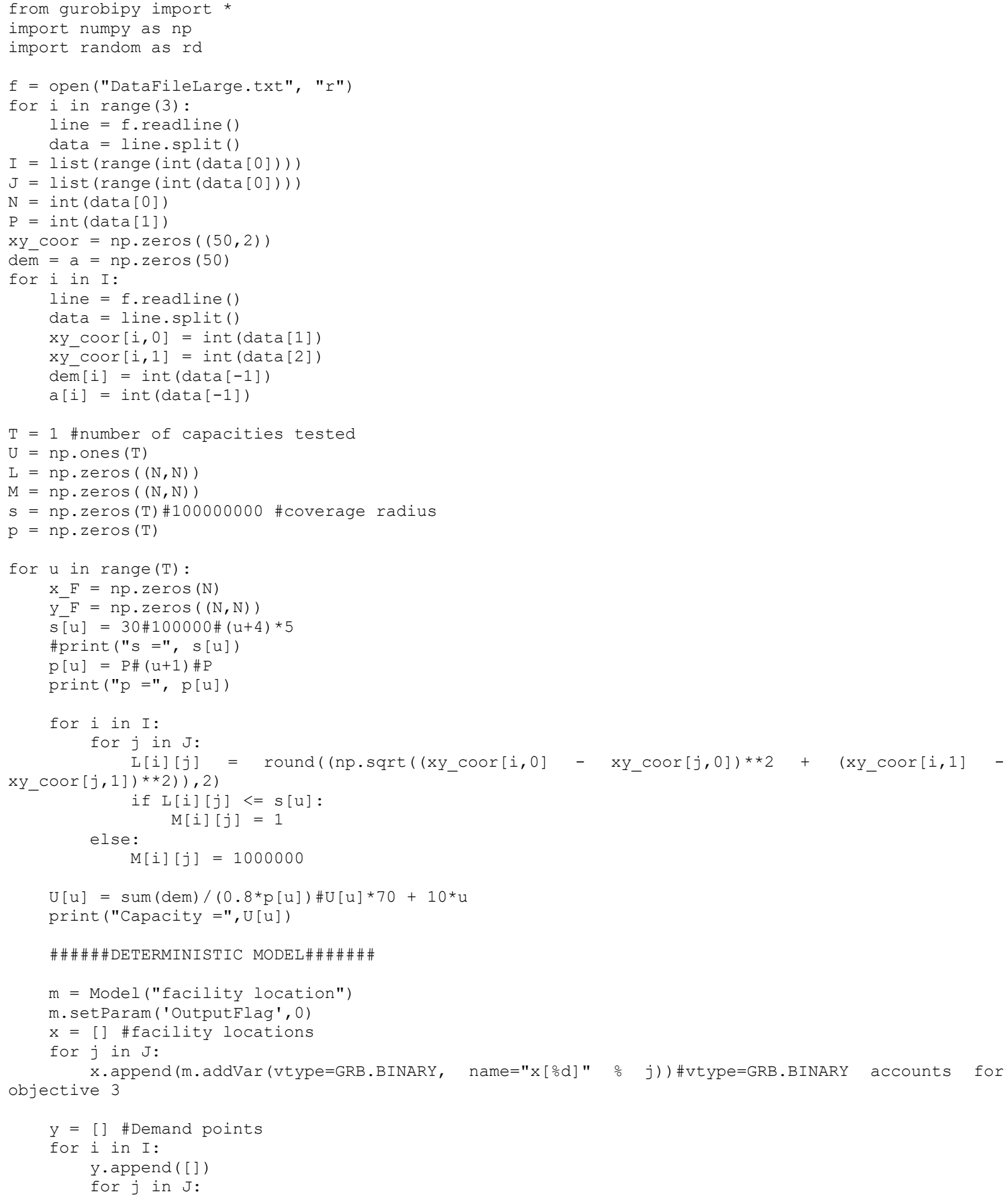




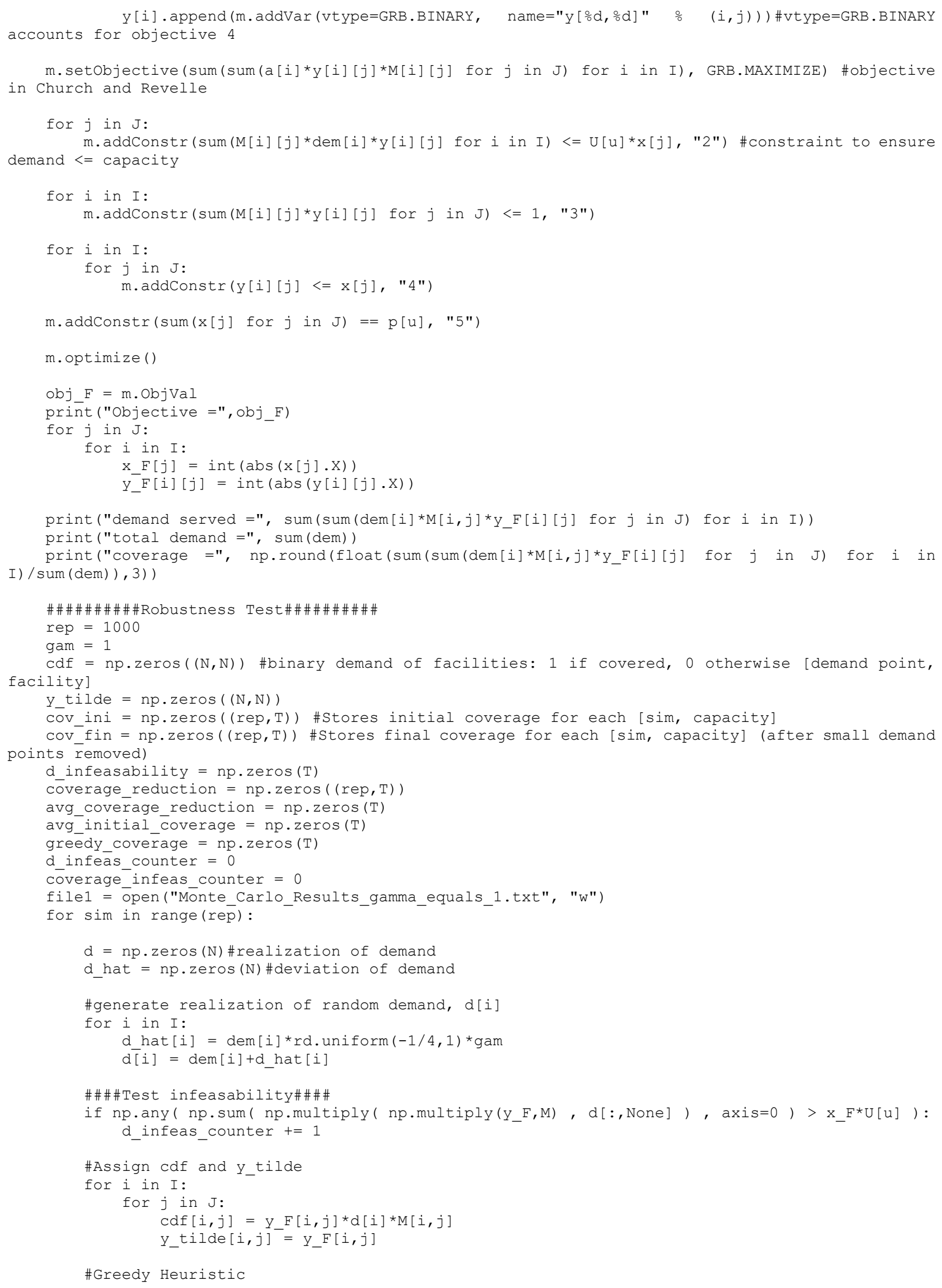


I) $/ \operatorname{sum}(\mathrm{dem}) \overline{)}$

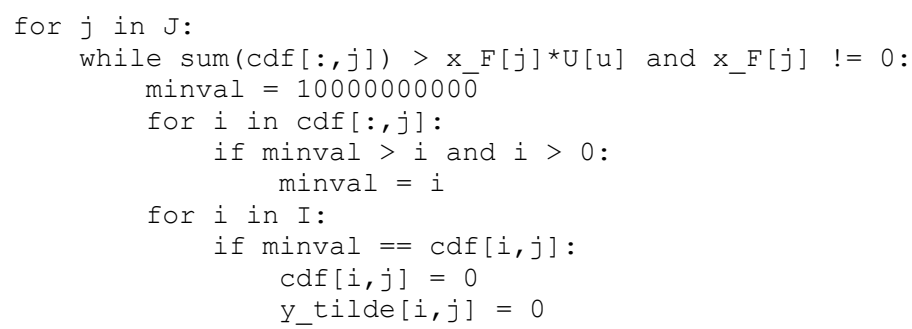

\#To show greedy heuristic is effective

\subsubsection{Robust}

from gurobipy import *

import numpy as np

import random as rd

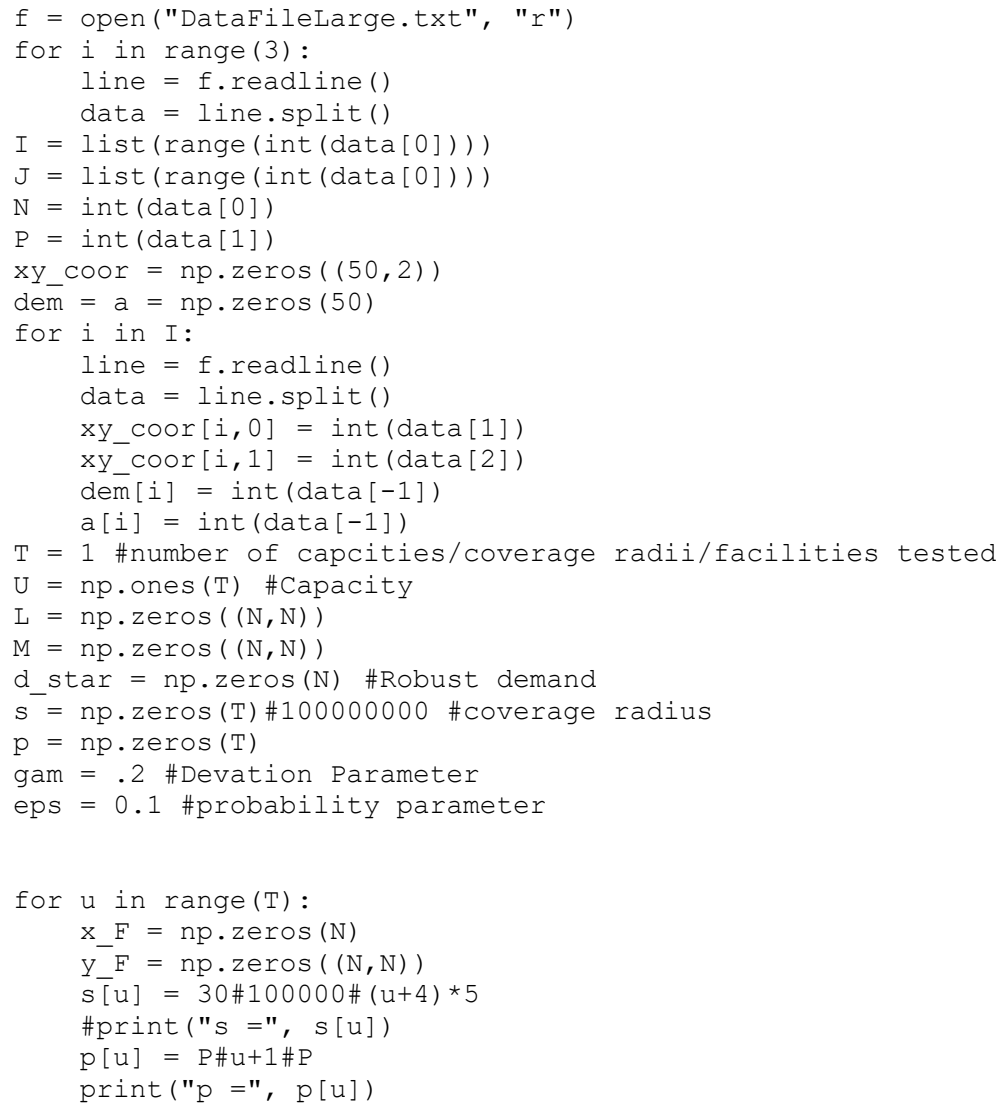




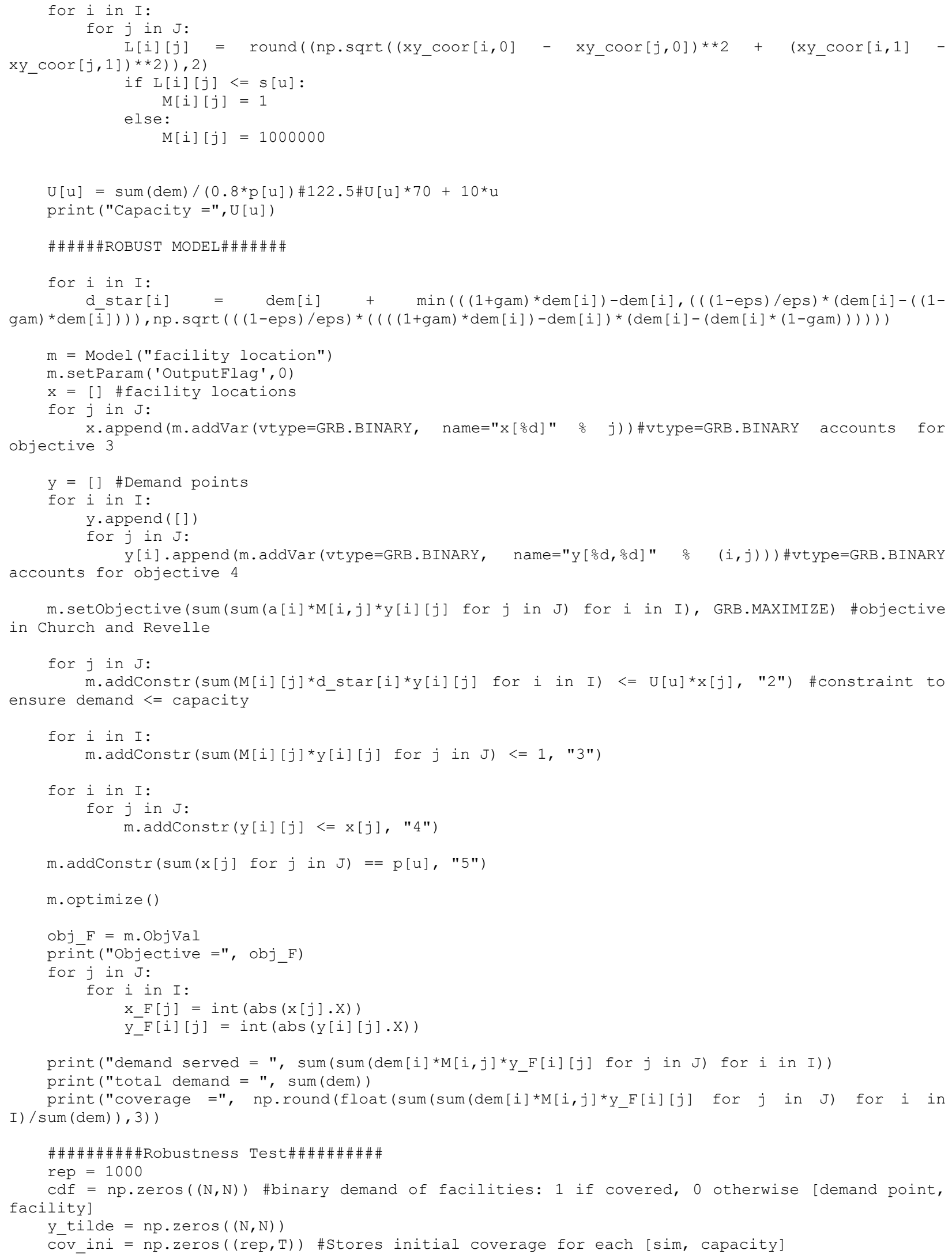


cov_fin $=n p . z e r o s((r e p, T))$ \#Stores final coverage for each [sim, capacity] (after small demand points removed)

d_infeasability $=n p \cdot \operatorname{zeros}(\mathrm{T})$

coverage_reduction $=n p \cdot \operatorname{zeros}((r e p, T))$

avg_coverage_reduction $=\mathrm{np}$.zeros $(\mathrm{T})$

avg_initial_coverage $=\mathrm{np}$.zeros $(\mathrm{T})$

greedy_coverage $=$ np.zeros $(T)$

d infeas counter $=0$

coverage_infeas_counter $=0$

filel = open ("Röbust Monte Carlo Results gamma equals 1.txt", "w")

for sim in range(rep):

$\mathrm{d}=\mathrm{np} \cdot \operatorname{zeros}(\mathrm{N})$ \#realization of demand

d_hat $=$ np.zeros $(\mathrm{N})$ \#deviation of demand

\#generate realization of random demand, dii]

for $i$ in $I$ :

d_hat $[i]=\operatorname{dem}[i] *$ rd.uniform $(-1 / 4,1) *$ gam

$d[i]=\operatorname{dem}[i]+d$ hat $[i]$

\#\#\#\#Test infeasability\#\#\#\#

if np.any ( np.sum( np.multiply( np.multiply(y_F,M), d[:,None]), axis=0 ) > $\left.x_{-} F^{\star} U[u]\right)$ : d_infeas_counter $+=1$

\#Assign cdf and y_tilde

for $i$ in $I$ :

for $j$ in $J$ :

$\operatorname{cdf}[i, j]=y F[i, j] \star d[i]$

$y_{-}$tilde $[i, j]^{-}=y_{-} F[i, j]$

\#Greedy Heuristic

for $j$ in $\mathrm{J}$ :

while sum $(\operatorname{cdf}[:, j])>x_{-} F[j] \star U[u]$ and $x_{-} F[j] !=0$ :

minval $=10000000$

for $i$ in $\operatorname{cdf}[:, j]$ :

if minval $>i$ and $i>0$ :

minval $=i$

for $i$ in $I$ :

if minval $==\operatorname{cdf}[i, j]$ :

$\operatorname{cdf}[i, j]=0$

y_tilde $[i, j]=0$

\#To show greedy Heuristic is effective

for $j$ in $J$ :

if $\operatorname{sum}\left(\operatorname{cdf}[:, j]>x_{-} F[j] * U[u]\right):$

coverage_infeas_counter $+=1$

I) $/ \operatorname{sum}(\operatorname{dem}) \bar{I}$

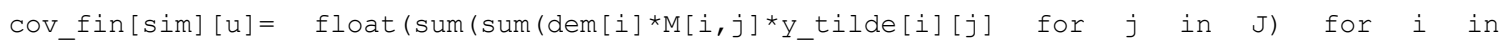

coverage reduction $[\operatorname{sim}][u]=$ float $\left(\operatorname{sum}\left(\operatorname{sum}\left(\operatorname{dem}\left[i{ }^{\star} M[i, j]^{*} \mathrm{Y} F[i][j]\right.\right.\right.\right.$ for $j$ in $\left.J\right)$ for $i$ in

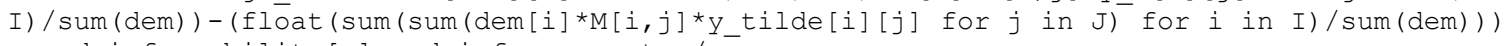

d_infeasability $[u]=d_{\text {_infeas_counter/rep }}$

cōverage_infeasability ${ }^{-}=$coverage_infeas_counter/rep

print("infeasability =", str(d_infeasability[u]))

print ("greedy coverage =", np.round(float (sum(cov_fin[sim] [u] for sim in range $(r e p)) / r e p), 3)$ )

print("coverage reduction $=", \quad n p . r o u n d(f l o a t(s u m(c o v e r a g e$ reduction[sim] [u] for sim in range (rep)) / rep), 3) )

print ( "\#\#\#\#\#\#\#\#\#\#\#\#\#\#\#\#\#\#\#\#\#\#\#")

\subsection{Python code for Minimum Cost FLP}

\subsubsection{Deterministic}

from gurobipy import *

import numpy as np

import random as rd

\# 1040444.375 


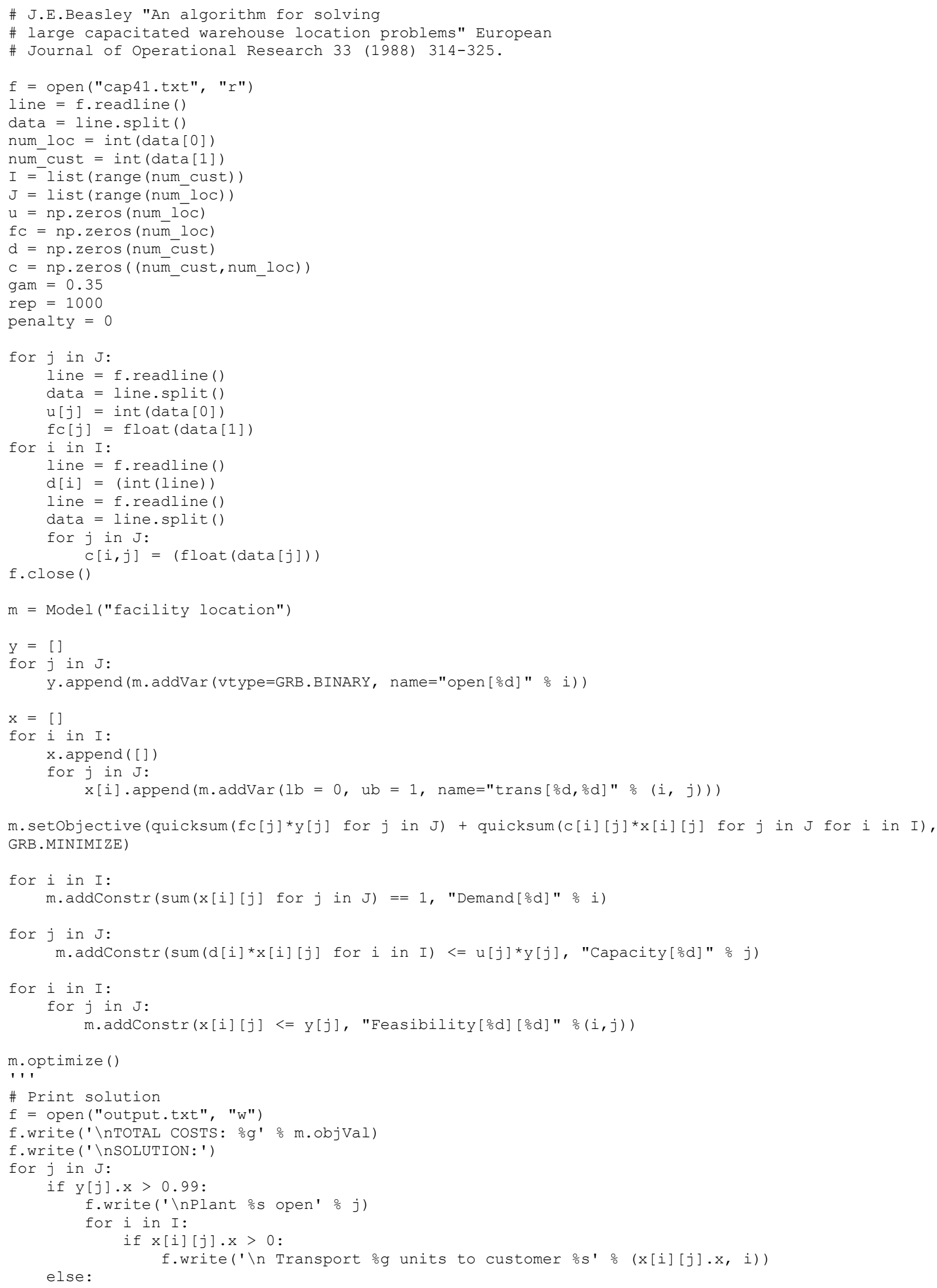




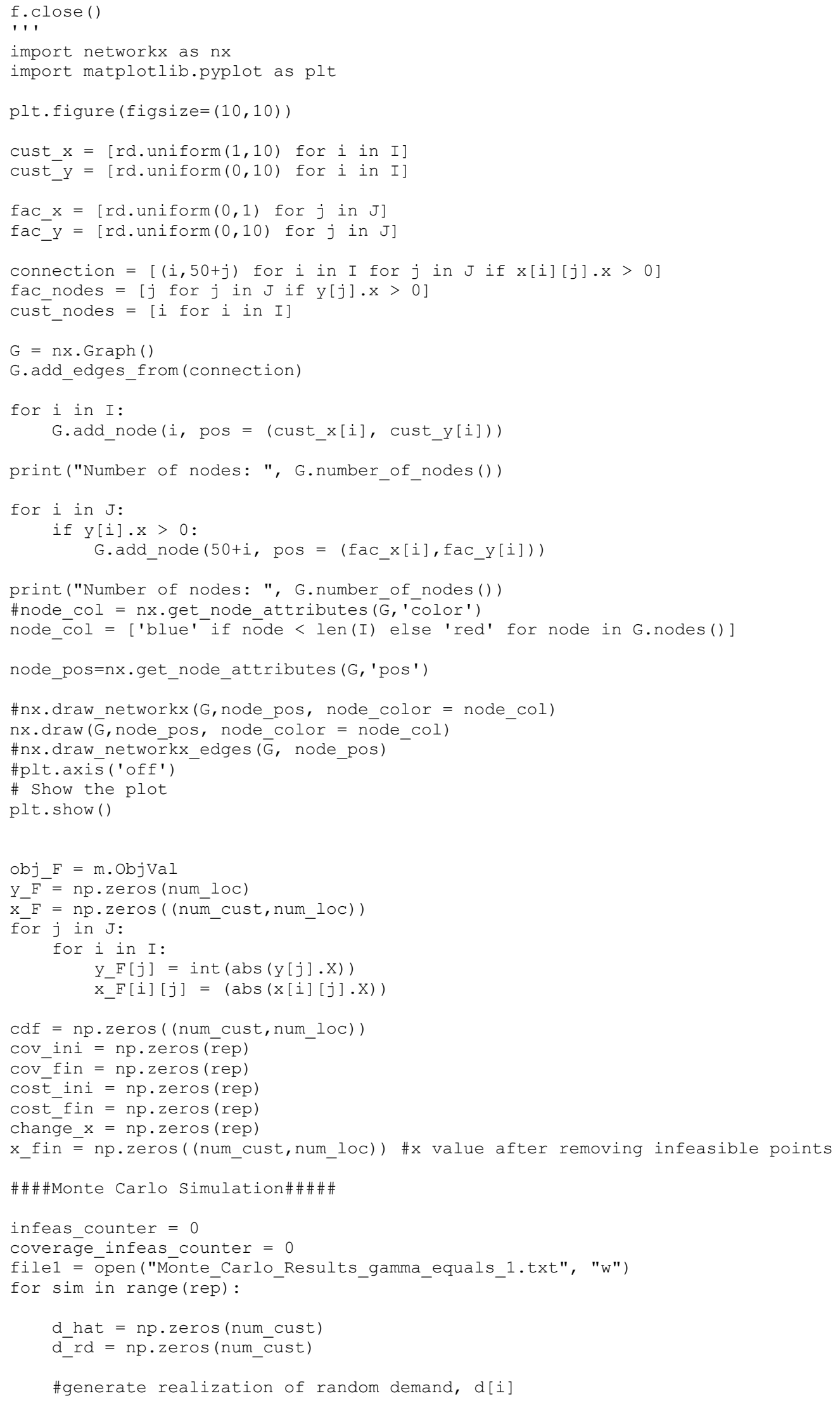




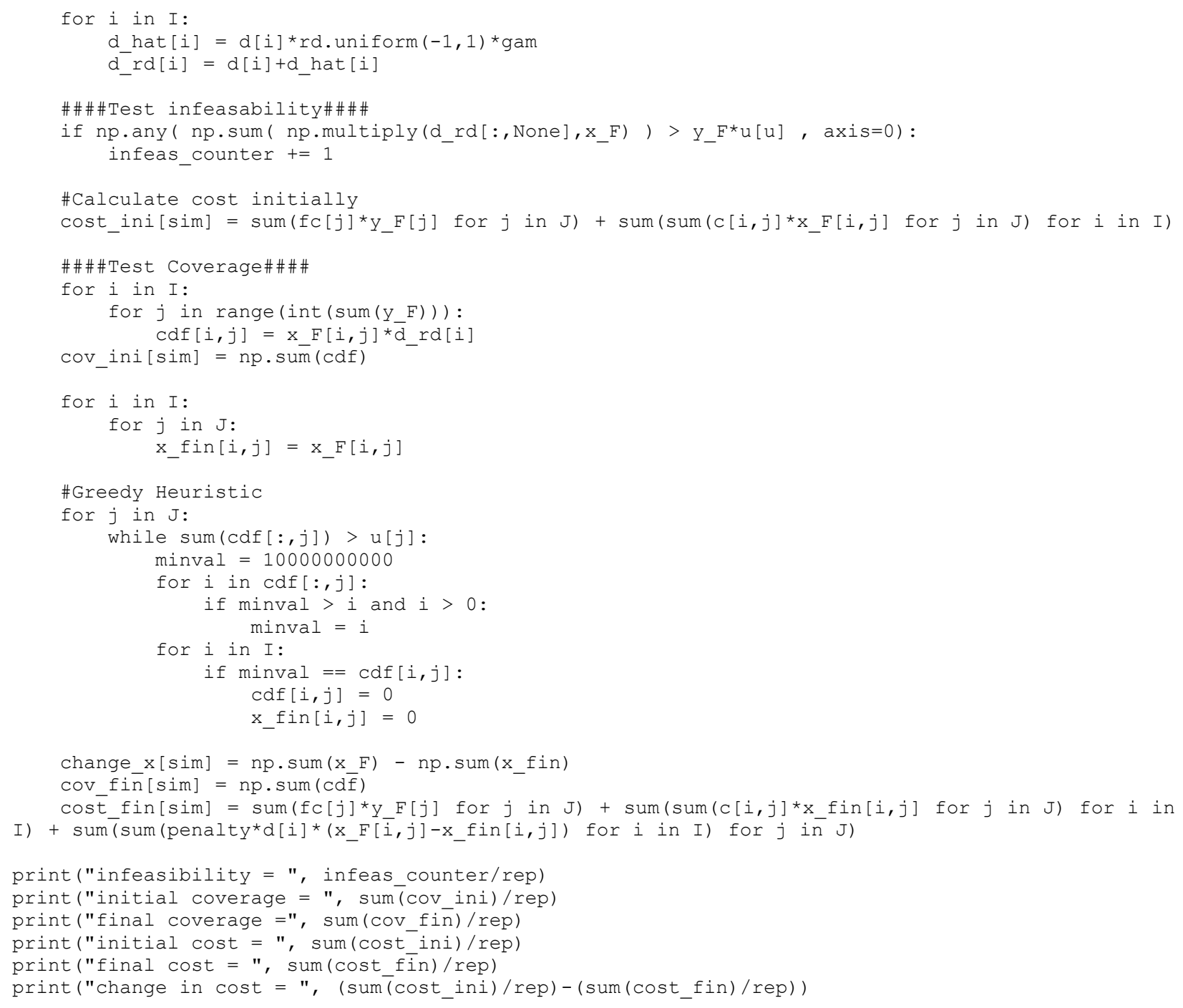

\subsubsection{Robust}

from gurobipy import *

import numpy as np

import random as rd

\# 1040444.375

\# J.E.Beasley "An algorithm for solving

\# large capacitated warehouse location problems" European

\# Journal of Operational Research 33 (1988) 314-325.

$\mathrm{f}=$ open ("cap41.txt", "r")

line $=$ f.readline ()

data $=$ line.split ()

num_loc $=$ int $($ data $[0])$

num_cust $=$ int $(\operatorname{data}[1])$

$I={ }^{-}$list (range (num cust))

$J=$ list (range (num_loc))

$\mathrm{u}=\mathrm{np} \cdot$ zeros (num lōo) \#capcity

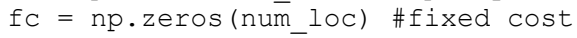

$\mathrm{d}=\mathrm{np}$.zeros (num cust) \#demand

d_star = np.zeros (num_cust) \#robust demand

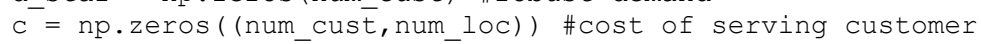

gamma $=0.2$

epsilon $=0.05$ 


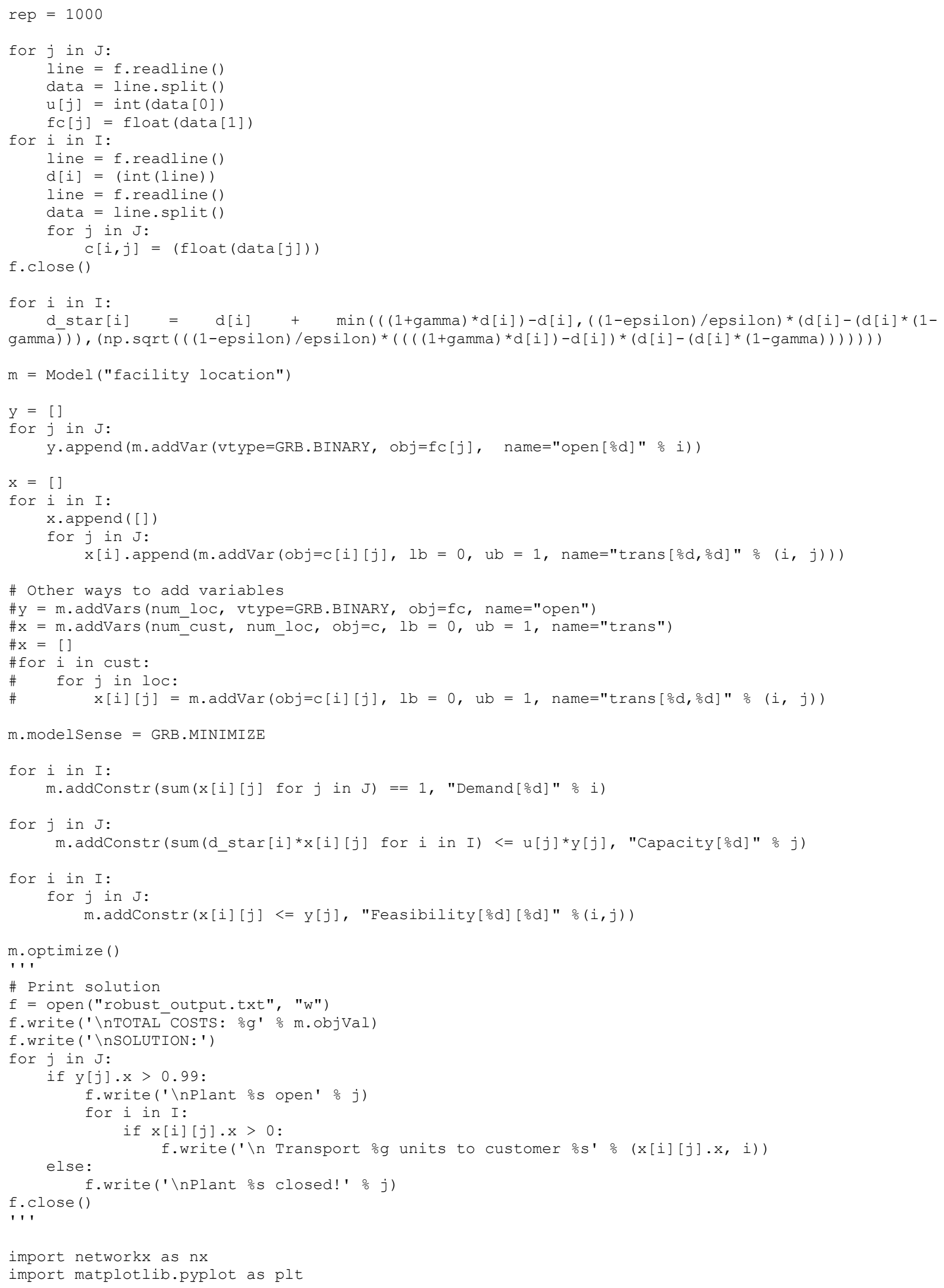




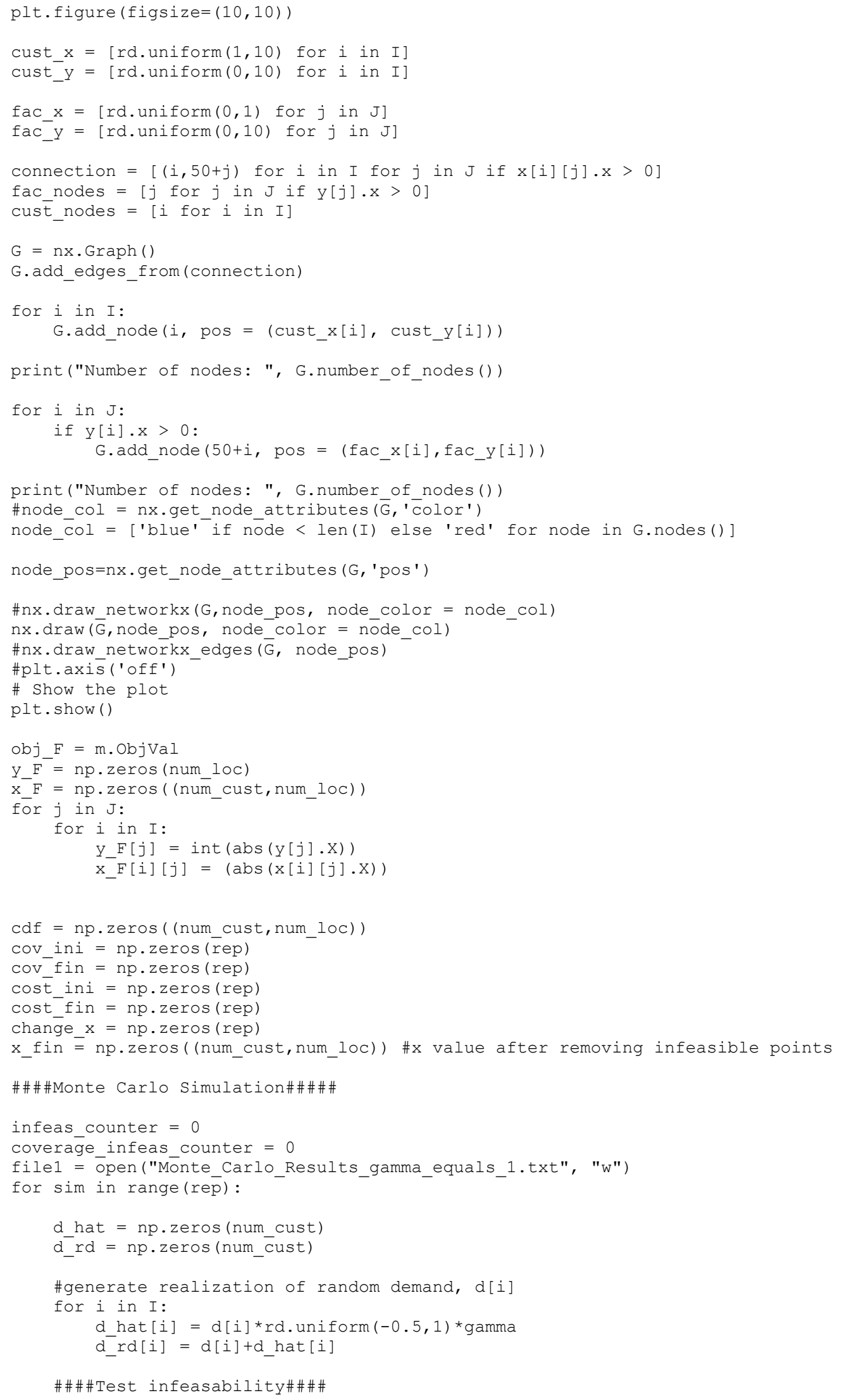




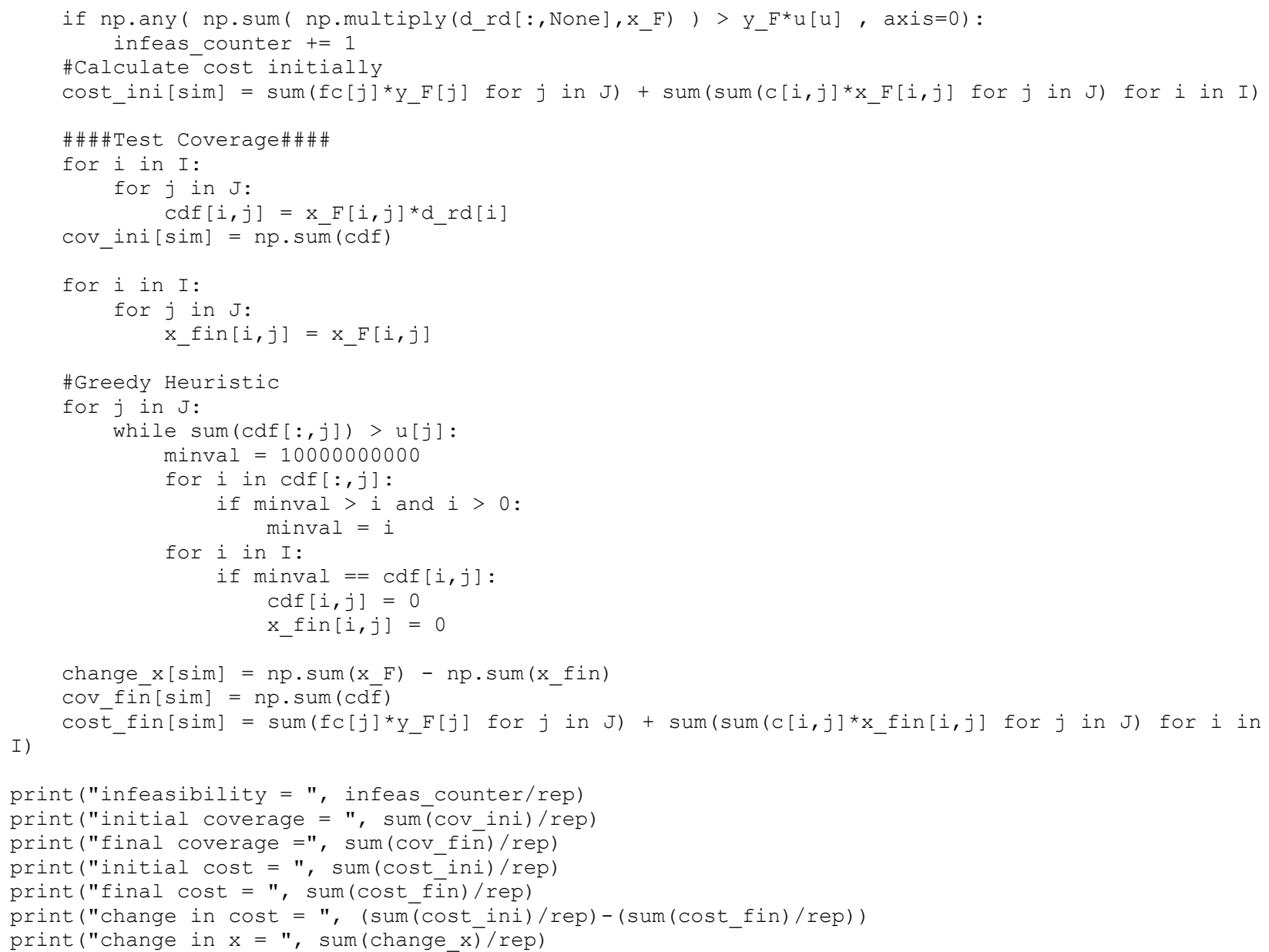

